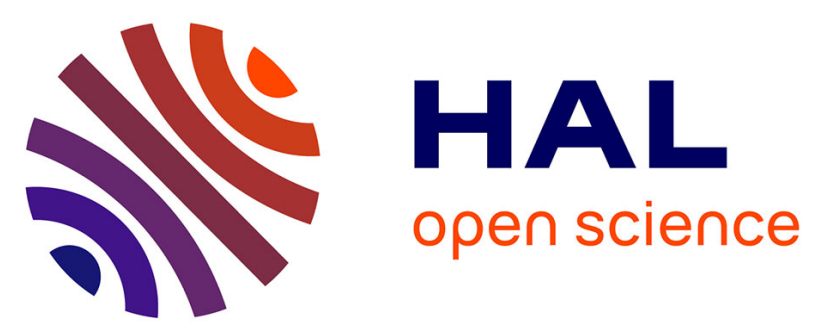

\title{
Effect of 3d/4p Mixing on 1s2p Resonant Inelastic X-ray Scattering: Electronic Structure of Oxo-Bridged Iron Dimers
}

Thomas Kroll, Michael L Baker, Samuel A Wilson, Marcus Lundberg, Amélie Juhin, Marie-Anne Arrio, James J Yan, Leland B Gee, Augustin Braun, Tsu-Chien Weng, et al.

\section{To cite this version:}

Thomas Kroll, Michael L Baker, Samuel A Wilson, Marcus Lundberg, Amélie Juhin, et al.. Effect of 3d/4p Mixing on 1s2p Resonant Inelastic X-ray Scattering: Electronic Structure of Oxo-Bridged Iron Dimers. Journal of the American Chemical Society, 2021, 143, pp.4569 - 4584. 10.1021/jacs.0c11193 . hal-03400963

\section{HAL Id: hal-03400963 https://hal.science/hal-03400963}

Submitted on 1 Nov 2021

HAL is a multi-disciplinary open access archive for the deposit and dissemination of scientific research documents, whether they are published or not. The documents may come from teaching and research institutions in France or abroad, or from public or private research centers.
L'archive ouverte pluridisciplinaire HAL, est destinée au dépôt et à la diffusion de documents scientifiques de niveau recherche, publiés ou non, émanant des établissements d'enseignement et de recherche français ou étrangers, des laboratoires publics ou privés. 


\section{Effect of 3d/4p Mixing on 1s2p Resonant Inelastic X-ray Scattering: Electronic Structure of oxo-bridged Iron Dimers}

Thomas Kroll, ${ }^{* \dagger, \ddagger}$ Michael L. Baker, ${ }^{\dagger}$ Samuel A. Wilson, ${ }^{\dagger}$ Marcus Lundberg, ${ }^{\dagger}, \S$ Amélie Juhin," Marie-Anne Arrio," James J. Yan, ${ }^{\dagger}$ Leland B. Gee, ${ }^{\dagger}$ Augustin Braun, ${ }^{\dagger}$ Tsu-Chien Weng, ${ }^{\ddagger} \perp$ Dimosthenis Sokaras, ${ }^{\ddagger}$ Britt Hedman, ${ }^{*}$, Keith O. Hodgson, ${ }^{*, \dagger, \ddagger}$ and Edward I. Solomon ${ }^{*, \dagger, \ddagger}$

$\dagger$ Department of Chemistry, Stanford University, Stanford, CA 94305, USA $\ddagger$ Stanford Synchrotron Radiation Lightsource, SLAC National Accelerator Laboratory, Stanford University, Menlo Park, CA 94025, USA

IDepartment of Chemistry, The University of Manchester, Manchester M13 9PL, United Kingdom

$\S$ Department of Chemistry - Angström, Uppsala University, SE-751 20 Uppsala, Sweden

|| Institut de Minéralogie, de Physique des Matériaux et de Cosmochimie (IMPMC),

Sorbonne Universités, UMR CNRS 7590, UPMC Univ Paris 06, Muséum National d'Histoire Naturelle, IRD UMR206, 4 Place Jussieu, F-75005 Paris, France $\perp$ Current address: School of Physical Science and Technology, ShanghaiTech University, Shanghai 201210, China

E-mail: tkroll@slac.stanford.edu; hedman@slac.stanford.edu; hodgson@slac.stanford.edu; edward.solomon@stanford.edu 


\begin{abstract}
$1 s 2 p$ resonant inelastic x-ray scattering ( $1 s 2 p$ RIXS) has proven successful in the determination of the differential orbital covalency (DOC, the amount of metal vs. ligand character in each $d$ molecular orbital) of highly covalent centrosymmetric iron environments including heme models and enzymes. However, many reactive intermediates have non-centrosymmetric environments, e.g. the presence of strong metal-oxo bonds, which results in the mixing of metal $4 p$ character into the $3 d$ orbitals. This leads to significant intensity enhancement in the metal K-pre-edge and as shown here, the associated $1 s 2 p$ RIXS features, which impact their insight into electronic structure. Binuclear oxo bridged high spin Fe(III) complexes are used to determine the effects of $4 p$ mixing on $1 s 2 p$ RIXS spectra. In addition to developing the analysis of $4 p$ mixing on K-edge XAS and $1 s 2 p$ RIXS data, this study explains the selective nature of the $4 p$ mixing that also enhances the analysis of L-edge XAS intensity in terms of DOC. These $1 s 2 p$ RIXS biferric model studies enable new structural insight from related data on peroxo bridged biferric enzyme intermediates. The dimeric nature of the oxo bridged Fe(III) complexes further results in ligand-to-ligand interactions between the Fe(III) sites and angle dependent features just above the pre-edge that reflect the superexchange pathway of the oxo bridge. Finally, we present a methodology that enables DOC to be obtained when L-edge XAS is inaccessible and only $1 s 2 p$ RIXS experiments can be performed as in many metalloenzyme intermediates in solution.
\end{abstract}




\section{Introduction}

The chemical and physical properties of the active sites in many proteins and enzymes are often determined by first-row transition metal ions. Spectroscopic tools to enhance the understanding of the metal $3 d$ orbitals and their covalent ligand interactions are therefore of high importance. X-ray spectroscopy involves creating a core-hole and thus offers an element specific method for obtaining detailed insight into the electronic structure. ${ }^{1}$ This is a particular advantage in highly covalent environments such as heme metallo-enzymes, where the metal signal can be obscured by the strong heme background in other spectroscopic methods. Soft X-ray absorption spectroscopy (XAS) in particular transition metal $\mathrm{L}_{2,3^{-}}$ edge XAS, which studies the metal $2 p \rightarrow 3 d$ transitions, has proven to be invaluable in determining detailed electronic structure information including the ligand-to-metal (LMCT) and metal-to-ligand (MLCT) charge transfer character (i.e. donor and acceptor bonding) for each metal $3 d$ orbital (i.e. the differential orbital covalency, DOC). ${ }^{2-4}$ As soft X-rays usually require high vacuum conditions partially due to the $e^{-}$detection over photon detection and low penetration depth, L-edge XAS is often not suitable for metallo-proteins and enzymes. Hard X-ray $1 s 2 p$ resonant inelastic X-ray scattering ( $1 s 2 p$ RIXS) measurements have a high penetration depth and do not require an UHV sample environment. This RIXS experiment involves resonantly exciting a $1 s$ electron into unoccupied $3 d$ orbitals, followed by a photon emitted by the decay of a $2 p$ electron to fill the $1 s$ core hole. ${ }^{5}$ Thus both the photon-in and photon-out energies are appropriate for metallo-enzymes in solution. The resulting $1 s 2 p$ RIXS final states have the same configurations as the L-edge XAS final states, but with different selection rules than in direct L-edge XAS. ${ }^{6}$ In a previous study on cytochrome c, $1 s 2 p$ RIXS only data were used to determine the DOC of the ferrous and ferric protein

sites starting from well described heme model complexes. ${ }^{7}$ Characteristic features in the $1 s 2 p$ RIXS planes were identified that reflect the main influences of the electronic structure changes associated with going from the heme model complex with two axial imidazole ligands to the heme protein with axial methionine and histidine ligands. ${ }^{7}$ 
The intensity and shape of the K-pre-edge is largely dominated by the local ligation around the transition metal ion. In a complex with close to inversion symmetry the $1 s \rightarrow 3 d$ pre-edge transitions are electric quadrupole allowed and relatively weak. In the absence of an inversion center, metal $4 p$ character can mix into the $3 d$ orbitals and contribute electric dipole allowed intensity in the K-pre-edge. Since this is approximately 100 times stronger than $1 s \rightarrow 3 d$ transitions, only a few percent of $4 p$ mixing can perturb and dominate the pre-edge spectral shape. ${ }^{8} 1 s 2 p$ RIXS planes are constructed by moving the incident energy through the pre-edge region for fixed emission energies or the other way around. By integrating along the energy transfer direction (i.e. the vertical axis in the RIXS plane that is the energy difference between the photon in and the photon out), the full K-edge XAS is obtained. Therefore, $1 s 2 p$ RIXS planes will also be affected by a loss of inversion. Importantly, due to the 2-dimensional nature of the RIXS planes, this electric dipole contribution to the pre-edge region can also provide information on the electronic structure that complements the insight available from direct $2 p \rightarrow 3 d$ L-edge XAS.

Mixing of $4 p$ character into the $3 d$ orbitals can occur in two ways. One is a direct interaction due to the non-centrosymmetric components of the ligand field, which corresponds to a direct matrix element between the metal $3 d$ and $4 p$ orbitals. ${ }^{9}$ In this ligand field theory method, the amount of $4 p$ character mixed into the $3 d$ orbitals is independent of the ligandmetal covalent overlaps and has been used without the explicit inclusion of LMCT in the Hamiltonian. ${ }^{10-13}$ The alternative and more efficient mechanism of $p / d$ mixing involves their covalent interactions with the ligands. This requires the explicit inclusion of the ligand orbitals in the Hamiltonian resulting in a $3 \times 3$ matrix:

\begin{tabular}{c|ccc} 
& $|F e 4 p\rangle$ & $|L 2 p\rangle$ & $|F e 3 d\rangle$ \\
\hline$\langle F e 4 p|$ & $\Delta^{4 p}$ & $T^{4 p}$ & 0 \\
$\langle L 2 p|$ & $T^{4 p}$ & 0 & $T$ \\
$\langle F e 3 d|$ & 0 & $T$ & $\Delta$
\end{tabular}


where the metal $3 d$ (at an energy $\Delta$ ) mixes with the ligand $p$ orbitals through their overlap $T$, and the same ligand orbital further overlaps the metal $4 p$ orbital, $T^{4 p}$ (at an energy $\Delta^{4 p}$ ). ${ }^{14}$ In this study, the LMCT $p / d$ mixing method is included as a direct result of the covalent bonds (additional details are given in the Supporting Information).

Many key biological active sites in particular the high valent $\mathrm{Fe}(\mathrm{IV})=\mathrm{O}$ heme and nonheme enzyme intermediates and their model complexes ${ }^{15,16}$ have no inversion center at the Fe site leading to intense K-pre-edge XAS features. This is also the case for the biferric peroxo and high-valent biferryl intermediates found in nonheme diiron enzymes and their model complexes. ${ }^{17-19} 1 s 2 p$ RIXS offers a unique opportunity to study these complexes due to its element selectivity and sensitivity to DOC. In this study, we determine the effects of $3 d / 4 p$ mixing on the spectral shape of $1 s 2 p$ RIXS 2D maps of binuclear high-spin ferric $\mathrm{Fe}(\mathrm{III})$ complexes having approximate $O_{h}$ and $C_{4 v}$ local symmetry. We have chosen high-spin $\mathrm{Fe}(\mathrm{III})$ systems as these are well understood from L-edge $\mathrm{XAS}^{2}$ and provide a good starting point to understand the $4 p$ mixing effects of an Fe(III)-oxo bond. In addition, they have key structural features anticipated to be present in biferric peroxo enzyme intermediates. ${ }^{20}$ Three Fe-dimer complexes were studied: The hydroxo bridged $\mathrm{Fe}_{2}-\mathrm{OH}-\left(\mathrm{HBpz}_{3}\right)_{2}(\mu \text {-ac })_{2}$ complex $\left(\mathrm{hbpz}_{3}=\text { tri-1-pyrazolylborate, } \mu \text {-ac }=\text { carboxylate bridge }\right)^{21}$ has a quasi-octahedral local Fe environment and serves as a centrosymmetric reference (see Figure 1 A). Deprotonation of the hydroxo bridge to form a biferric oxo-bridge (with the same ligand environment, $\mathrm{Fe}_{2}$ oxo- $\left(\mathrm{HBpz}_{3}\right)_{2}(\mu \text {-ac })_{2}$, Figure $\left.1 \mathrm{~B}\right)$, leads to short Fe-O bond lengths (1.96 to $1.79 \AA$ in $\mu$-OH and $\mu$-oxo, respectively). This eliminates the approximate inversion symmetry at the Fe reducing the local symmetry to approximately $C_{4 v}$. Both complexes have an Fe-O-Fe angle of $\sim 124^{\circ}$. A second oxo-bridged biferric complex is included in this study, where the ligand system is changed to hedta, and the Fe-O-Fe bond angle increases to $165^{\circ}$ (Figure $1 \mathrm{C}$ ), but keeps the same Fe-oxo bond lengths, the high-spin configuration and approximate local $C_{4 v}$ symmetry.

In this study, the L-edge, K-edge XAS spectra and $1 s 2 p$ RIXS 2D maps of all three 


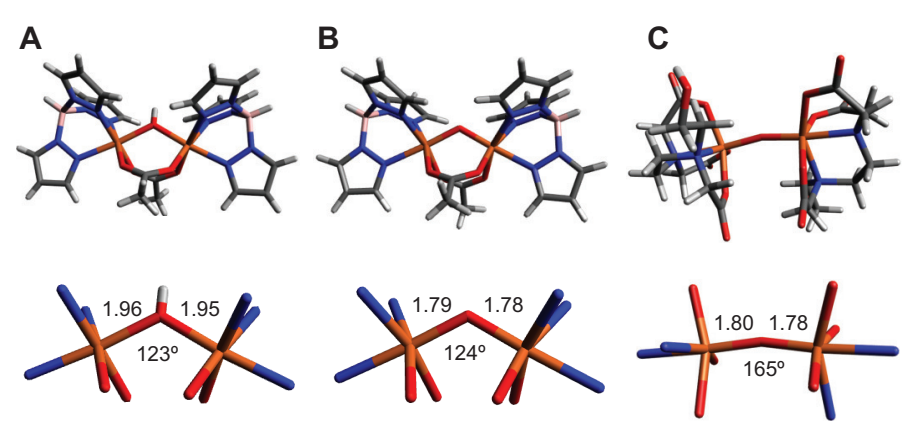

Figure 1: Crystal structures of the three model compounds $\mathrm{Fe}_{2}-\mathrm{OH}-\left(\mathrm{HBpz}_{3}\right)_{2}(\mu-\mathrm{ac})_{2} \quad(\mathrm{~A})$, $\mathrm{Fe}_{2}$-oxo- $\left(\mathrm{HBpz}_{3}\right)_{2}(\mu \text {-ac })_{2}$ (B) and $\mathrm{Fe}_{2}$-oxo-hedta 2 (C).

complexes are analyzed to extract the DOC using a valence bond configuration interaction (VBCI) mulitplet model to fit L-edge XAS and RIXS data. ${ }^{2,6,7}$ The $2 p$ core-hole in the L-edge XAS and $1 s 2 p$ RIXS final states leads to strong $2 p$ spin-orbit coupling and $2 p$ - $3 d$ Coulomb repulsion that influence the relative intensities and shapes of the $L_{3^{-}}$and $L_{2}$-edge and the $1 s 2 p$ RIXS maps and must be included in the multiplet analysis to quantitatively estimate covalency (i.e. DOC). Advantages (and disadvantages) of the strong electric dipole intensity contributions from $4 p$ mixing to the RIXS planes are determined by comparing cuts through the $1 s 2 p$ RIXS map to L-edge and K-edge XAS data. For many biological systems, L-edge XAS cannot be measured and only $1 s 2 p$ RIXS would be available. Therefore the determination of the covalent ligand-metal bonding in terms of DOC from $1 s 2 p$ RIXS planes alone is further developed. This study also provides new insight into the molecular orbital origin of the $4 p$ mixing in $\mathrm{Fe}=\mathrm{O}$ systems and explains the dominant $3 d\left(z^{2}\right) / 4 p(z)$ intensity contribution found experimentally. The DOC allows access to the covalent mixing in the oxo- $d \pi$ and oxo- $d \sigma$ orbitals, which are the frontier molecular orbitals (FMOs) in metal-oxo systems that play a key role in many reactions.

To fully analyze the data, we take two complementary approaches. One where we use the VBCI multiplet model to fit the experimental data and extract bonding information in terms of DOC from experiment. This is then compared to Mulliken populations from DFT calculations enabling the correlation of the experimental RIXS data to the DFT calculations. This complementary approach also provides insight into dimer specific covalent 
mixing through inter-oxo bridged ligand-ligand interactions and angle dependent inter Fe transitions reflecting oxo superexchange pathways

Using the advanced insight from the analysis of the full $1 s 2 p$ RIXS maps of the hydroxoand oxo-bridged model complexes as references, we also gain new insight into the geometric structures of several peroxo bridged biferric enzyme intermediates, in particular when an oxo bridge is also present that would greatly impact reactivity.

\section{Experimental and Computational Methods}

Sample Preparation: Crystalline samples of $\mathrm{Fe}_{2}-\mathrm{OH}-\left(\mathrm{HBpz}_{3}\right)_{2}(\mu-\mathrm{ac})_{2}, \mathrm{Fe}_{2}$-oxo- $\left(\mathrm{HBpz}_{3}\right)_{2}(\mu-$ ac) $)_{2}$, and $\mathrm{Fe}_{2}$-oxo-hedta 2 were prepared following standard procedures. ${ }^{21-24}$

Experimental Methods: In this study, two experimental methods have been employed: soft X-ray L-edge XAS and $1 s 2 p$ RIXS. These experiments have been performed at the Stanford Synchrotron Radiation Lightsource (SSRL) at beam lines 10-1 (L-edge XAS) and 6-2 (1s2p RIXS). ${ }^{25}$ Experimental details on both methods are given in the Supporting Material. All data sets (L-edge XAS, $1 s 2 p$ RIXS) have been measured at least twice to ensure reproducible and reliable data. Special attention has been paid to avoid photo damage by moving over the sample and illuminating each sample spot for only a short amount of time.

All $1 s 2 p$ RIXS planes are normalized to unity at the edge-jump allowing for a direct comparison with K-edge XAS results. The pre-edge in a $1 s 2 p$ RIXS experiment includes the tail of the intense $1 s \rightarrow 4 p$ transitions at approximately $15 \mathrm{eV}$ above the pre-edge. This leads to an increase of the pre-edge background and complicates the direct comparison of L-edge data with constant incident energy (CIE, i.e. vertical) cuts through the RIXS plane. In order to estimate the RIXS pre-edge intensity from the tail of the rising-edge, the full RIXS planes were fit to extract the true pre-edge region as described previously. ${ }^{7,26}$ 
Charge Transfer Multiplet Simulations: To simulate both L-edge and RIXS X-ray spectra, charge transfer multiplet calculations were performed. ${ }^{27-30}$ The calculations were performed in the $C_{4 v}$ crystal field symmetry for all three complexes. Covalent mixing of ligand character into unoccupied $3 d$ orbitals was modeled using the valence bond configuration interaction (VBCI) approach. Ligand-to-metal charge transfer (LMCT) is introduced via a $d^{n+1} \underline{L}(\underline{L}=$ ligand hole $)$ configuration that is mixed into the $d^{n}$ configuration in the ground state. LMCT charge transfer channels were included through the overlap parameters $T\left(B_{1}\right)$, $T\left(A_{1}\right), T(E)$, and $T\left(B_{2}\right)$ with the corresponding ligand-to-metal charge transfer energy $\Delta .4 p$ mixing was included by the introduction of an additional ligand-to-metal(4p) charge transfer $\left(\mathrm{LM}_{4 \mathrm{p}} \mathrm{CT}\right)$ configuration. Here, the $\mathrm{LM}_{4 \mathrm{p}} \mathrm{CT}$ channels were included via $T\left(A_{1}\right)\left(4 \mathrm{p}_{\mathrm{z}} / 3 \mathrm{~d}_{\mathrm{z}^{2}}\right)$ and $T(E)\left(4 \mathrm{p}_{\mathrm{x}, \mathrm{y}} / 3 \mathrm{~d}_{\mathrm{xz}, \mathrm{yz}}\right)$ as allowed in $\mathrm{C}_{4 \mathrm{v}}$ symmetry at an energy $\Delta^{4 p}$. Details including a description of the model and the calculation of absolute absorption intensities normalized to the edge jump are given in the Supporting Material. The metal-ligand covalency for each of the symmetry blocks (i.e. the DOC) was calculated using the projection method. ${ }^{2}$ Here, each $3 d$ hole has $100 \%$ metal character. Upon ligand-to-metal covalent mixing, the metal character decreases (and ligand character in the $3 d$ orbitals increases).

Initial VBCI fits to the L-edge XAS spectra were obtained using a trust region reflective non-linear least squares fitting approach from a series of 100 sets of start parameters that were randomly generated. From these, the best parameter set was determined by their refinement of simultaneously fitting to both the L-edge XAS and $1 s 2 p$ RIXS data (see Supporting Information for more details).

Density Functional Theory Calculations: All calculations were carried out using the ORCA quantum chemistry program, version 4.0.0.2. ${ }^{31}$ For all calculations, the non-optimized crystal structures of the three complexes ${ }^{21-23}$ were used. The B3LYP functional ${ }^{32}$ was employed with standard polarized triple-zeta basis set def2-TZVPP 33 and auxiliary basis set $\operatorname{def} 2 / \mathrm{J},{ }^{34}$ and the more flexible $\mathrm{CP}(\mathrm{PPP})$ basis set $^{35}$ on the central di-iron ions. A dense 
integration grid (grid4), and tight convergence criteria were enforced.

For TD-DFT derived K pre-edge XAS spectra, ${ }^{36-40}$ the application of scalar relativistic corrections (ZORA) ${ }^{41,42}$ did not lead to a better description and has thus been omitted, as found previously. ${ }^{38}$ For all XAS calculations, electric dipole, electric quadrupole and magnetic dipole contributions are included. Calculated K pre-edge spectra were visualized using the ORCA_MAPSPC module with a Gaussian broadening of $1.0 \mathrm{eV}$. For transitions that are electric-dipole allowed, the electric-quadrupole and magnetic dipole contributions become origin dependent. ${ }^{43-45}$ To account for this, the origin adjusted method (ABSQ) using a minimization procedure that guarantees the fastest convergence of the multipole expansion was used, which gave similar results as the origin independent method (ABSOI) $)^{43,46}$ in the energy range of interest. A constant energy shift of $151.5 \mathrm{eV}$ was applied to the calculated spectra. Mulliken population analyses ${ }^{47}$ were performed using the QMForge Suite. ${ }^{48}$

\section{Results and Analysis}

Three sets of data were obtained for the three complexes: Fe L-edge and K-edge XAS, and $1 s 2 p$ RIXS. In this section, the L-edge XAS data are presented together with their charge transfer multiplet simulations and the resulting DOC. This is then compared to the results of DFT calculations. The K-edge is presented both from K-edge XAS and from high-resolution constant emission energy (CEE) cuts in the pre-edge and the extended pre-edge region of the RIXS plane and analyzed using TD-DFT calculations. The constant incident energy (CIE) cuts are then presented, connected to the L-edge XAS and analyzed with the multiplet model. Finally, given a well defined relevant model complex we show that the $1 s 2 p$ RIXS plane alone can be used to define the DOC of an intermediate in solution. 


\section{L-edge XAS and DOC}

Soft X-ray L-edge XAS directly probes the unoccupied Fe $3 d$ orbitals via electric dipole allowed $2 \mathrm{p} \rightarrow 3 \mathrm{~d}$ transitions and therefore experimentally quantitatives the covalency (by the decrease in $d$ character and therefore intensity due to bonding). L-edge XAS for $\mathrm{Fe}_{2}$ $\mathrm{OH}-\left(\mathrm{HBpz}_{3}\right)_{2}(\mu \text {-ac })_{2}, \mathrm{Fe}_{2}$-oxo- $\left(\mathrm{HBpz}_{3}\right)_{2}(\mu \text {-ac })_{2}$, and $\mathrm{Fe}_{2}$-oxo-hedta 2 are shown in Figure 2 A-C (black), respectively.

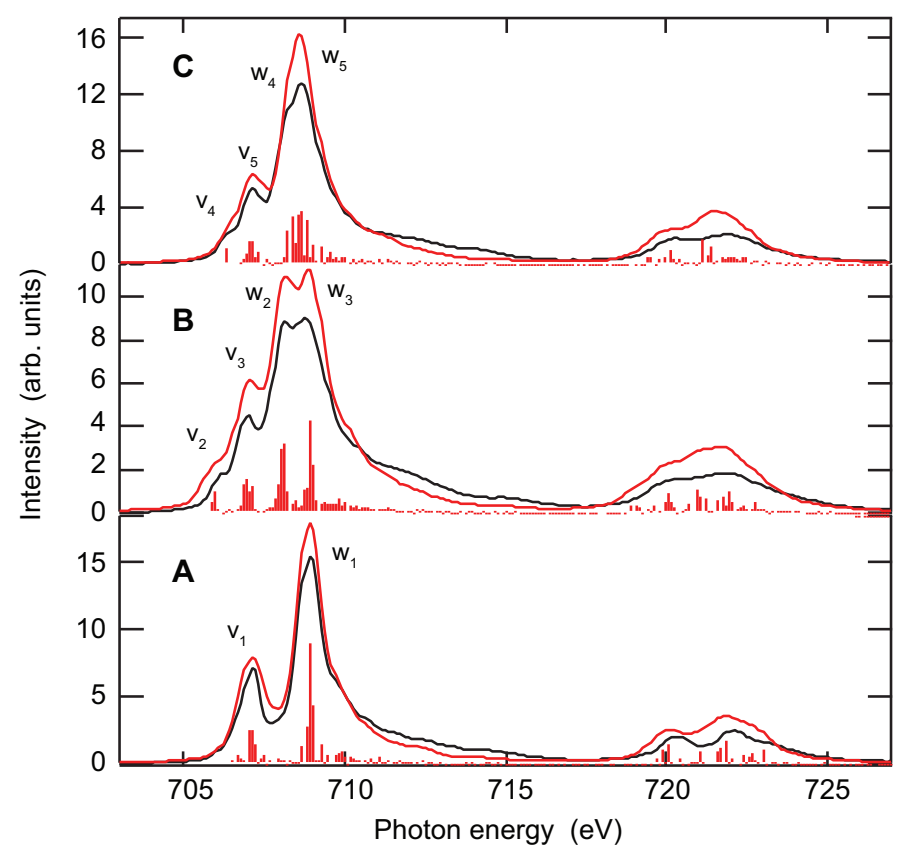

Figure 2: Experimental L-edge XAS together with their charge transfer multiplet simulations of $\mathrm{Fe}_{2}-\mathrm{OH}-\left(\mathrm{HBpz}_{3}\right)_{2}(\mu \text {-ac })_{2}(\mathrm{~A}), \mathrm{Fe}_{2}$-oxo- $\left(\mathrm{HBpz}_{3}\right)_{2}(\mu \text {-ac })_{2}(\mathrm{~B})$, and $\mathrm{Fe}_{2}$-oxo-hedta 2 (C). Experimental spectra are shown in black, while simulations are shown in red.

The $\mathrm{Fe}_{2}-\mathrm{OH}-\left(\mathrm{HBpz}_{3}\right)_{2}(\mu \text {-ac) })_{2}$ L-edge XAS spectrum (Figure $\left.2 \mathrm{~A}\right)$ is very similar to those of typical high spin $O_{h} \mathrm{Fe}(\mathrm{III})$ complexes. ${ }^{2}$ For high spin ferric $\mathrm{L}_{3}$-edges, the low energy intensity at $707.1 \mathrm{eV}\left(\mathrm{v}_{1}\right)$ originates predominantly from transitions into $\mathrm{d} \pi$ orbitals $\left(t_{2 g^{-}}\right.$ like) with a small amount of $\mathrm{d} \sigma$ ( $e_{g}$-like) character, while the intensity around $708.9 \mathrm{eV}\left(\mathrm{w}_{1}\right)$ contains similar $\mathrm{d} \pi$ and $\mathrm{d} \sigma$ contributions to the intensity (mixed by the $3 d-2 p$ exchange). ${ }^{49}$

Upon going from an $\mathrm{OH}$ to an oxo bridge (Figure $2 \mathrm{~A}$ to $\mathrm{B}$ and $\mathrm{C}$ ), the L-edge XAS spectra change reflecting the change in electronic structure due to the shortened Fe-oxo bonds (by $\sim 0.2 \AA$ ). ${ }^{21-23}$ The shortened Fe-O(bridge) bond length in the oxo dimer reduces 
the local geometric structure from quasi $O_{h}$ to $C_{4 v}$. As a result, the low and high energy peaks broaden due to the energy splitting of the $t_{2} \pi$ (into $b_{2}\left(d_{x y}\right)$ and $e\left(d_{x z, y z}\right)$ ) and $e \sigma$ (into $b_{1}\left(d_{x^{2}-y^{2}}\right)$ and $\left.a_{1}\left(d_{z^{2}}\right)\right)$ degeneracies.

The $\mathrm{Fe}_{2}-\mathrm{OH}-\left(\mathrm{HBpz}_{3}\right)_{2}(\mu \text {-ac })_{2}$ peak at $707.2 \mathrm{eV}\left(v_{1}\right)$ incident energy splits into two well separated features at 706.1 and $707.0 \mathrm{eV}$ for $\mathrm{Fe}_{2}$-oxo- $\left(\mathrm{HBpz}_{3}\right)_{2}(\mu-\mathrm{ac})_{2}\left(\mathrm{v}_{2}\right.$ and $\left.\mathrm{v}_{3}\right)$, and 706.3 and $707.2 \mathrm{eV}$ for $\mathrm{Fe}_{2}$-oxo-hedta $2\left(\mathrm{v}_{4}\right.$ and $\left.\mathrm{v}_{5}\right)$. The main peak at $708.9 \mathrm{eV}\left(w_{1}\right)$ for $\mathrm{Fe}_{2}-\mathrm{OH}-$ $\left(\mathrm{HBpz}_{3}\right)_{2}(\mu \text {-ac })_{2}$ splits into two well separated peaks at 708.1 and $707.7 \mathrm{eV}$ for $\mathrm{Fe}_{2}$-oxo$\left(\mathrm{HBpz}_{3}\right)_{2}(\mu-\mathrm{ac})_{2}\left(\mathrm{w}_{2}\right.$ and $\left.\mathrm{w}_{3}\right)$, and into a main peak at $708.7 \mathrm{eV}$ and a shoulder at $\sim 708.2 \mathrm{eV}$ for $\mathrm{Fe}_{2}$-oxo-hedta $\left(\mathrm{w}_{4}\right.$ and $\left.\mathrm{w}_{5}\right)$. Due to the shorter Fe-O(bridge) bond length, the $z^{2}$ level shifts to higher energies, while the $x y$ level should be at lowest energy. Quantitatively, the precise energy of these splittings are strongly influenced by the $p d$ and $d d e^{-}-e^{-}$Coulomb repulsion and exchange. ${ }^{50,51}$

The absolute integrated intensity of a normalized and background subtracted Fe L-edge XAS spectrum reflects the total metal $3 d$ character of the unoccupied valence orbitals. ${ }^{52,53}$ For the three biferric complexes, the integrated intensities are: $47 \pm 3$ units $\left(\mathrm{Fe}_{2}-\mathrm{OH}-\right.$ $\left.\left(\mathrm{HBpz}_{3}\right)_{2}(\mu \text {-ac })_{2}\right), 41 \pm 8$ units $\left(\mathrm{Fe}_{2}\right.$-oxo- $\left.\left(\mathrm{HBpz}_{3}\right)_{2}(\mu \text {-ac })_{2}\right)$ and $45 \pm 7$ units $\left(\mathrm{Fe}_{2}\right.$-oxo-hedta 2$)$. They represent similar metal $3 d$ characters within error, with the trends of an increased covalent mixing (i.e. decreased intensity, therefore lower metal $d$ character) upon deprotonation of the Fe-OH bridge (with the same $\left(\mathrm{HBpz}_{3}\right)_{2}(\mu \text {-ac })_{2}$ ligand environment) and a decreased covalency (increased metal $d$ character) upon exchanging the non-oxo ligand environment $\left(\operatorname{HBpz}_{3}(\mu-\mathrm{ac})_{2}\right.$ to hedta $)$.

While the total intensity is given by the ground state, the spectral shape is determined by the final states that are reached via the $2 p \rightarrow 3 d$ electric dipole transitions. ${ }^{53}$ To interpret the experimental spectra, simulations using charge transfer multiplet calculations are necessary that take the $3 d / 2 p$-core-hole interaction properly into account. ${ }^{50}$ Figure $2 \mathrm{~A}-\mathrm{C}$ (red curves) shows the best fits of $\mathrm{Fe}_{2}-\mathrm{OH}-\left(\mathrm{HBpz}_{3}\right)_{2}(\mu-\mathrm{ac})_{2}, \mathrm{Fe}_{2}$-oxo- $\left(\mathrm{HBpz}_{3}\right)_{2}(\mu \text {-ac })_{2}$, and $\mathrm{Fe}_{2}$-oxo-hedta 2 L-edge spectra, respectively, using multiplet calculations with valence bond 
Table 1: Summary of L-edge Integrated Intensities, DOC Simulation Results, and Molecular Orbital Calculations. Note that the value for $\mathrm{Fe}(e)$ is per orbital and the $e$ set carries twice this amount.

\begin{tabular}{|c|c|c|c|c|c|c|c|c|c|c|c|}
\hline & \multirow[b]{2}{*}{ intensity } & \multicolumn{5}{|c|}{ L-edge $+1 s 2 p$ RIXS covalency (\%) } & \multicolumn{5}{|c|}{ DFT covalency (\%) } \\
\hline & & $\mathrm{Fe}\left(\mathrm{b}_{2}\right)$ & $\mathrm{Fe}(\mathrm{e})$ & $\mathrm{Fe}\left(\mathrm{b}_{1}\right)$ & $\mathrm{Fe}\left(\mathrm{a}_{1}\right)$ & total & $\mathrm{Fe}\left(\mathrm{b}_{2}\right)$ & $\mathrm{Fe}(\mathrm{e})$ & $\mathrm{Fe}\left(\mathrm{b}_{1}\right)$ & $\mathrm{Fe}\left(\mathrm{a}_{1}\right)$ & total \\
\hline $\mathrm{Fe}_{2}-\mathrm{OH}-\left(\mathrm{HBpz}_{3}\right)_{2}(\mu-\mathrm{ac})_{2}$ & $47 \pm 3$ & 88 & 88 & 74 & 74 & 413 & 84 & 88 & 74 & 74 & 408 \\
\hline $\mathrm{Fe}_{2}$-oxo- $\left(\mathrm{HBpz}_{3}\right)_{2}(\mu-\mathrm{ac})_{2}$ & $41 \pm 8$ & 89 & 80 & 76 & 67 & 392 & 86 & 79 & 78 & 68 & 390 \\
\hline $\mathrm{Fe}_{2}$-oxo-hedta 2 & $45 \pm 7$ & 91 & 84 & 77 & 69 & 405 & 88 & 81 & 76 & 68 & 394 \\
\hline
\end{tabular}

configuration interaction (VBCI) included. Details on the parameters used are given in the Supporting Information. Note that these best fits result from the simultaneous analysis of the L-edge XAS (Figure 2) and $1 s 2 p$ RIXS data (vide infra). All spectral features and trends are well described including the additional ligand field broadening and splittings for the oxo bridged complexes.

For all three complexes, a high spin ground state is found consistent with experiment. By using the projection method on the ground state configurations, the metal $3 d$ character for each individual $3 d$ orbital, i.e. the differential orbital covalency (DOC), was determined. ${ }^{2}$ The resulting values from the total $d$ character from the L-edge XAS total intensity and from DFT Mulliken population analyses are given in Table 1.

The VBCI derived DOCs agree with the values found from DFT and with the total experimental intensity in showing an increased covalency (i.e. decreased metal $d$ character) in going from $\mathrm{Fe}_{2}$-OH-(HBpz $)_{2}(\mu \text {-ac })_{2}$ to $\mathrm{Fe}_{2}$-oxo- $\left(\mathrm{HBpz}_{3}\right)_{2}(\mu \text {-ac })_{2} \quad$ (413 to $\left.392 \%\right)$, as well as a decreased covalency (increased metal $d$ character) by changing the $\mathrm{Fe}_{2}$-oxo ligation from $\left(\mathrm{HBpz}_{3}\right)_{2}(\mu \text {-ac })_{2}$ to hedta 2 (392 to $\left.405 \%\right)$. Upon lowering the local symmetry from an OH to an oxo bridge, the orbitals having direct overlap with the bridging oxygen (local z-direction) are affected the most. The $e(d(x z / y z)(d \pi))$ metal character decreases from 88 to $80 \%$ per orbital and the $a_{1}\left(d\left(z^{2}\right)(d \sigma)\right)$ metal character decreases from 74 to $67 \%$. In going to the hedta $2 \mathrm{Fe}_{2}$-oxo dimer, the largest changes are observed in the $d \pi$ orbitals $\left(89 \rightarrow 91 \%\right.$ for $b_{2}$ and $80 \rightarrow 84 \%$ for $e$ ). Both, experiment and DFT calculations reflect these changes. 
2D RIXS planes
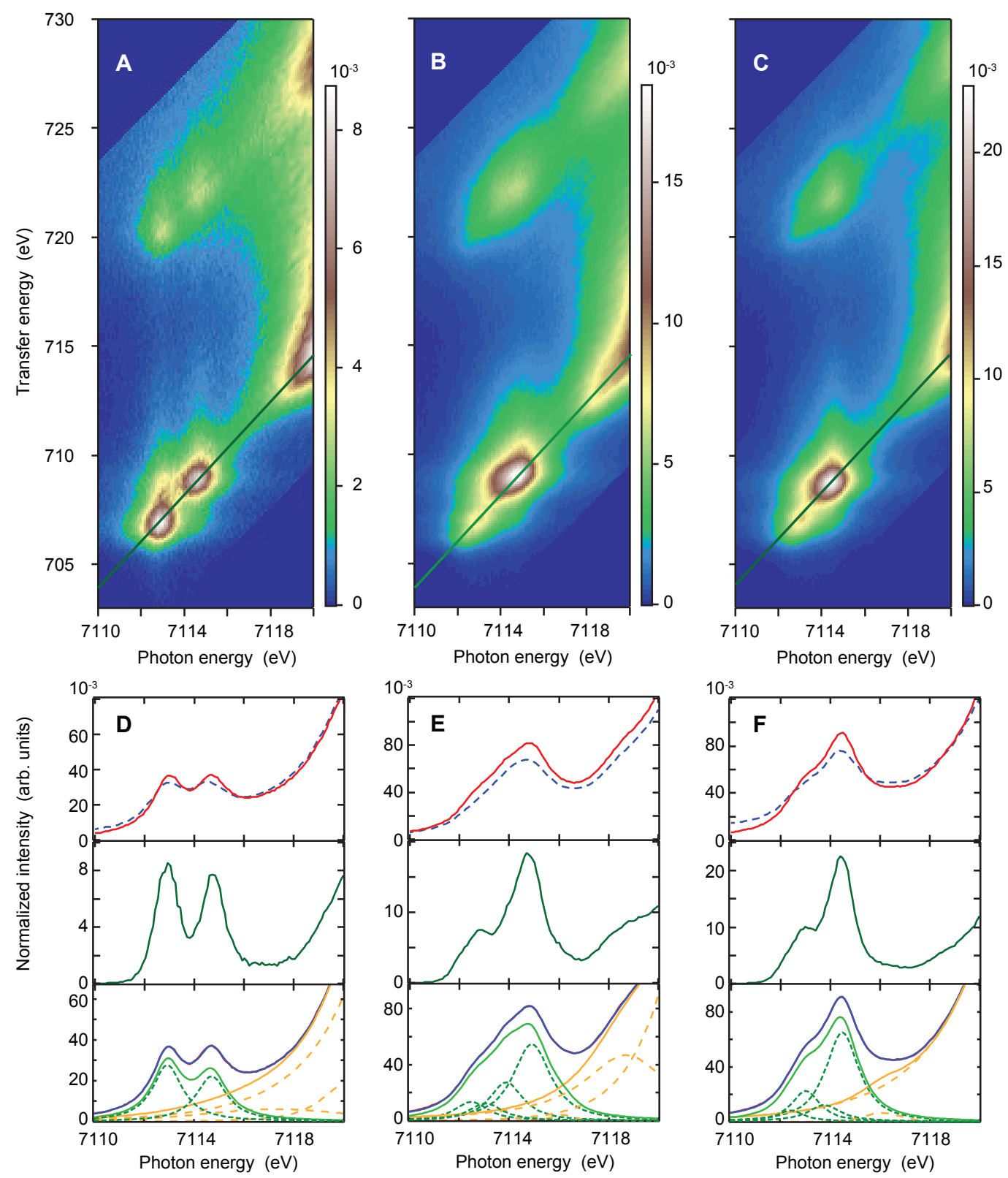

Figure 3: Experimental 1s2p RIXS planes of (A) $\mathrm{Fe}_{2}-\mathrm{OH}-\left(\mathrm{HBpz}_{3}\right)_{2}(\mu \text {-ac })_{2}$, (B) $\mathrm{Fe}_{2}$-oxo$\left(\mathrm{HBpz}_{3}\right)_{2}(\mu \text {-ac })_{2}$, and $(\mathrm{C}) \mathrm{Fe}_{2}$-oxo-hedta 2 . All spectra are normalized to the edge jump and plotted such that the maximum intensity is in the pre-edge region. (D)-(F) show the corresponding K-edge spectra from the integrated RIXS plane (red solid) and the direct Kedge XAS measurement (blue dashed, top), together with the CEE cut (green solid, middle). The direct K-edge data are adapted from Ref. 8. The bottom figures show the fits to the K-edge derived from the integrated RIXS planes. The resulting background subtracted preedge fit is shown in light green solid. 
In the $1 s 2 p$ RIXS process, a $1 s$ electron is excited into unoccupied $3 d$ orbitals with potential $4 p$ character mixed in via electric quadrupole and electric dipole transition moments, respectively. This absorption process probes the unoccupied density of states with the corresponding incident energy being plotted along the horizontal axis in the 2D maps (Figure 3). In the electric dipole $2 p \rightarrow 1 s$ emission process, plotted as the transfer energy (difference between the energy of the photon in and the photon out, vertical axis) in the $1 s 2 p$ RIXS planes, the occupied density of states is probed through the Coulomb and exchange interaction of the $2 p$ core-hole with the $3 d$ electrons. Figure 3 shows the $1 s 2 p$ RIXS planes normalized to the edge-jump of $\mathrm{Fe}_{2}-\mathrm{OH}-\left(\mathrm{HBpz}_{3}\right)_{2}(\mu \text {-ac })_{2} \quad(\mathrm{~A}), \mathrm{Fe}_{2}$-oxo- $\left(\mathrm{HBpz}_{3}\right)_{2}(\mu$ ac) $)_{2}$ (B), and $\mathrm{Fe}_{2}$-oxo-hedta 2 (C). The maximum of the color scale is set to the maximum intensity in the individual pre-edge region. All three complexes show pre-edge intensities between $\sim 7112-7116 \mathrm{eV}$ clearly separated from the rising edge (visible at $>7119 \mathrm{eV}$ ) in the 2D presentation.

For $\mathrm{S}=5 / 2$ high spin systems in quasi $O_{h}$ environments, two main transitions to the intermediate state $(1 s \rightarrow 3 d)$ are expected into the $t_{2 g}$ and $e_{g}$ orbitals, ${ }^{8}$ while for the oxobridged systems in $C_{4 v}$ symmetry four transitions are expected due to the additional $C_{4 v}$ ligand field splitting $\left(t_{2 g}\left(\mathrm{O}_{\mathrm{h}}\right)\right.$ splits into $b_{2}, e$, and $e_{g}\left(\mathrm{O}_{\mathrm{h}}\right)$ into $\left.b_{1}, a_{1}\right)$. As expected, the $\mathrm{Fe}_{2^{-}}$ $\mathrm{OH}-\left(\mathrm{HBpz}_{3}\right)_{2}(\mu \text {-ac })_{2}$ RIXS map shows two separated pre-edge peaks with similar intensities at 7112.9 and $7114.6 \mathrm{eV}$. In going to the oxo-bridged complexes, the pre-edge intensity pattern changes. In both oxo bridged high spin ferric complexes, one dominant feature at $\sim 7114.8 \mathrm{eV}\left(\mathrm{Fe}_{2}\right.$-oxo- $\left(\mathrm{HBpz}_{3}\right)_{2}(\mu \text {-ac })_{2}$, Figure $\left.3 \mathrm{~B}\right)$ and $7114.4 \mathrm{eV}\left(\mathrm{Fe}_{2}\right.$-oxo-hedta 2 , Figure 3C) is visible together with a low intensity feature at lower energies. The high intensity of the $\sim 7114.8$ and $\sim 7114.4 \mathrm{eV}$ peaks emphasizes the relevance of the additional electric dipole intensity due to the $4 p$ character mixed in the $3 d$ orbitals.

A more detailed analysis is done by cuts through the 2D $1 s 2 p$ RIXS plane: Diagonal cuts through the RIXS plane along the constant emission energy (CEE) give K-edge like spectra with higher resolution than conventional K-edge XAS due to the long-lived $2 p$ core-hole final 
state $^{54,55}$ These will be analyzed in the next section (K-Edge and CEE Spectra). Vertical cuts along the transfer energy direction at constant initial energy (CIE) reach final states that are identical to those reached in direct L-edge XAS but with different selection rules. ${ }^{6}$ This allows a direct comparison of $1 s 2 p$ RIXS and L-edge XAS and will be presented in a later section (L-edge-like CIE cuts).

\section{K-Edge and CEE Spectra}

The Pre-Edge Region K-pre-edge XAS involves $1 s \rightarrow 3 d$ transitions into unoccupied valence orbitals. These spectra are shown in Figure 3 (D-F) as derived from the integrated RIXS map (red solid, top) and the direct K-edge XAS measurement (blue dashed, top). The $1 s 2 p$ RIXS derived K-edge aligns well with the direct K-edge XAS measurement taken from Ref 8 , including absolute intensities. The K-pre-edge spectrum of the approximately $O_{h}$ complex, $\mathrm{Fe}_{2}-\mathrm{OH}-\left(\mathrm{HBpz}_{3}\right)_{2}(\mu \text {-ac })_{2}$, resolves two peaks at $\sim 7113$ and $\sim 7114.5 \mathrm{eV}$, consistent with other high spin $d^{5}$ studies. ${ }^{8}$ Upon lowering the local symmetry to $C_{4 v}$ in the $\mu$-oxo bridged complexes (Figure 3E and F), the pre-edge intensity doubles due to additional mixing of $4 p$ character in the $3 d$ orbitals. The increased intensity is most pronounced in the higher energy region of the K-pre-edge at $\sim 7114.5 \mathrm{eV}$.

These observations become more prominent in the CEE cuts as shown in Figure 3D-F (green solid, middle). In high spin $\mathrm{S}=5 / 2 \mathrm{Fe}(\mathrm{III}) \mathrm{K}$-pre-edges the multiplet effects simplify to a one electron orbital description. ${ }^{8}$ Therefore, two main transitions are expected in $O_{h}$ symmetry and four transitions in $C_{4 v}$ due to the additional orbital splittings. As anticipated, the $\mathrm{Fe}_{2}-\mathrm{OH}-\left(\mathrm{HBpz}_{3}\right)_{2}(\mu \text {-ac })_{2}$ spectrum contains two CEE peaks, while four peaks are necessary to fit the CEE cuts of $\mathrm{Fe}_{2}$-oxo-hedta 2 and $\mathrm{Fe}_{2}$-oxo- $\left(\mathrm{HBpz}_{3}\right)_{2}(\mu \text {-ac })_{2}$ (see Figure $3 \mathrm{D}-\mathrm{F}$ bottom).

The full K-edges i.e. integrated $1 s 2 p$ RIXS planes, were fit with two (OH-bridged) and four peaks (oxo-bridged) in the pre-edge region together with a proper background. Note that relative intensities in the $\mathrm{CEE}$ cuts do not necessarily represent the correct intensity ratios 
due to off diagonal intensity contributions in the $1 s 2 p$ RIXS planes. The fits to the three complexes are shown in Figure 3D-F (bottom) and Figure S2 together with a description of the procedure to fit the data. For $\mathrm{Fe}_{2}-\mathrm{OH}-\left(\mathrm{HBpz}_{3}\right)_{2}(\mu-\mathrm{ac})_{2}$, the low energy peak at $7112.9 \mathrm{eV}$ accounts for $55 \%$ of the total pre-edge intensity, or 5.9 out of 10.7 total units of intensity normalized to the edge jump (Table 2).

Table 2: Results of a multiple peak fit to the K pre-edge of the three complexes. The energy position is given in $\mathrm{eV}$, while the intensity is given in absolute units.

\begin{tabular}{|c|c|c|c|c|c|}
\hline \multirow[b]{2}{*}{$\mathrm{Fe}_{2}-\mathrm{OH}-\left(\mathrm{HBpz}_{3}\right)_{2}(\mu-\mathrm{ac})_{2}$} & \multirow{2}{*}{$\begin{array}{c}\text { total } \\
\text { intensity } \\
10.7\end{array}$} & \multicolumn{2}{|c|}{ peak i } & \multicolumn{2}{|c|}{ peak ii } \\
\hline & & $\begin{array}{l}\text { energy } \\
7112.9\end{array}$ & $\begin{array}{c}\text { intensity } \\
5.9\end{array}$ & $\begin{array}{l}\text { energy } \\
7114.7\end{array}$ & $\begin{array}{c}\text { intensity } \\
4.8 \\
\end{array}$ \\
\hline & total & peak 1 & peak 2 & peak 3 & peak 4 \\
\hline & intensity & energy int. & energy int. & energy int. & energy int. \\
\hline $\mathrm{Fe}_{2}$-OxO- $\left(\mathrm{HBpz}_{3}\right)_{2}(\mu-\mathrm{ac})_{2}$ & 24.9 & 7112.5 & $7113.0 \quad 2.7$ & 7114.2 & 7115.0 \\
\hline $\mathrm{Fe}_{2}$-oxo-hedta 2 & 22.7 & 7112.4 & 7113.0 & 7113.8 & 7114.5 \\
\hline
\end{tabular}

In $\mathrm{Fe}_{2}$-oxo- $\left(\mathrm{HBpz}_{3}\right)_{2}(\mu \text {-ac })_{2}$ and $\mathrm{Fe}_{2}$-oxo-hedta 2 , the local symmetry lowers to $C_{4 v}$ due to the short Fe-O bridge bond, and $4 p$ character is mixed into the $3 d$ orbitals. $^{8,10-12,56,57}$ In $C_{4 v}$ symmetry, the $4 p(z)$ orbital is allowed to mix with the $3 d\left(z^{2}\right)\left(a_{1}\right)$ and the $4 p(x, y)$ orbitals are allowed to mix with the $3 d(x z, y z)(e)$. The mixing of $4 p$ character into the $3 d$ orbitals results in a $1 \mathrm{~s} \rightarrow 4 \mathrm{p}$ electric dipole contribution to the pre-edge. As a consequence, the total pre-edge intensities of the $\mathrm{Fe}_{2}$-oxo- $\left(\mathrm{HBpz}_{3}\right)_{2}(\mu \text {-ac })_{2}$ and $\mathrm{Fe}_{2}$-oxo-hedta 2 spectra increase by around a factor of two relative to the $\mathrm{Fe}_{2}-\mathrm{OH}-\left(\mathrm{HBpz}_{3}\right)_{2}(\mu \text {-ac })_{2}$ pre-edge intensity. In more detail, the spectral shapes of both $\mu$-oxo-bridged complexes are now dominated by an intense feature at higher energies, which is better resolved in the CEE cuts. The four peaks fit to the data (Figure 3 (bottom)) reveals that the highest energy peak at $\sim 7115 \mathrm{eV}$ gains considerable intensity, while the two lower energy peaks (peaks 1 and 2 in Table 2, the $b_{2}$ and $e$ components in $C_{4 v}$ ), which correlate to the first peak for the quasi octahedral $\mathrm{Fe}_{2}-\mathrm{OH}-\left(\mathrm{HBpz}_{3}\right)_{2}(\mu \text {-ac })_{2}$ complex, do not gain intensity. Therefore, only $a_{1}$ mixing occurs, even though $d \pi / p_{x, y}(e)$ mixing is allowed by group theory.

Estimating the amount of $4 p$ character mixed into the $3 d$ pre-edge of the experimental 
Table 3: Amount of $4 p$ character mixed into the unoccupied $3 d$ orbitals from a Mulliken analysis of the DFT single point calculations. The small values that are found in orbitals with $x$ and $y$ character originate from a deviation of the $C_{4 v}$ symmetry.

\begin{tabular}{lcccc}
\hline & \multicolumn{4}{c}{ Amount of $4 p$ character [\%] } \\
& $x y$ & $x z / y z$ & $x^{2}-y^{2}$ & $z^{2}$ \\
\hline $\mathrm{Fe}_{2}$-OH- $\left(\mathrm{HBpz}_{3}\right)_{2}(\mu-\mathrm{ac})_{2}$ & 0.3 & 0.2 & 0.3 & 0.5 \\
$\mathrm{Fe}_{2}$-oxo- $\left(\mathrm{HBpz}_{3}\right)_{2}(\mu-\mathrm{ac})_{2}$ & 0.3 & 0.8 & 0.3 & 2.0 \\
$\mathrm{Fe}_{2}$-oxo-hedta & 0.2 & 1.7 & 1.0 & 3.8 \\
\hline
\end{tabular}

spectrum was achieved by subtracting the estimated quadrupole contribution taken from the $\mathrm{Fe}_{2}$-OH- $\left(\mathrm{HBpz}_{3}\right)_{2}(\mu \text {-ac })_{2}$ K-edge data. ${ }^{8}$ The total intensities for $\mathrm{Fe}_{2}$-oxo-hedta 2 and $\mathrm{Fe}_{2}$ oxo- $\left(\mathrm{HBpz}_{3}\right)_{2}(\mu \text {-ac })_{2}$ are 22.7 and 24.9 units, respectively, while the $\mathrm{Fe}_{2}-\mathrm{OH}-\left(\mathrm{HBpz}_{3}\right)_{2}(\mu-$ ac) $)_{2}$ total intensity is 10.7 units. From the L-edge results in Table 1, $\mathrm{Fe}_{2}$-oxo-hedta 2 has 0.98 of the metal $d$ character of $\mathrm{Fe}_{2}-\mathrm{OH}-\left(\mathrm{HBpz}_{3}\right)_{2}(\mu \text {-ac })_{2}$ (i.e. $2 \%$ more covalent), while $\mathrm{Fe}_{2}$-oxo$\left(\mathrm{HBpz}_{3}\right)_{2}(\mu \text {-ac })_{2}$ has 0.95 of the metal $d$ character (i.e. $5 \%$ more covalent). Assuming that the K-pre-edge spectrum of $\mathrm{Fe}_{2}-\mathrm{OH}-\left(\mathrm{HBpz}_{3}\right)_{2}(\mu \text {-ac })_{2}$ consists purely of electric quadrupole transitions, the expected quadrupole intensities of $\mathrm{Fe}_{2}$-oxo-hedta 2 and $\mathrm{Fe}_{2}$-oxo- $\left(\mathrm{HBpz}_{3}\right)_{2}(\mu$ ac) $)_{2}$ are $\sim 10.5$ and $\sim 10.2$ units, respectively. This gives $\sim 12.2$ and $\sim 14.7$ units of intensity for the electric dipole contribution to the $\mathrm{Fe}_{2}$-oxo-hedta 2 and $\mathrm{Fe}_{2}$-oxo- $\left(\mathrm{HBpz}_{3}\right)_{2}(\mu \text {-ac })_{2}$ spectra, respectively. From these values, the percentage of $4 p$ mixing into the $3 d$ orbitals can be estimated. Following Westre et al. ${ }^{8}$ this pre-edge extracted intensity correlates to $6 \%$ and $7 \%$ $4 p$ mixing into the $3 d$ orbitals for $\mathrm{Fe}_{2}$-oxo-hedta 2 and $\mathrm{Fe}_{2}$-oxo- $\left(\mathrm{HBPz}_{3}\right)_{2}(\mu \text {-ac })_{2}$, respectively.

From a Mulliken analysis of the DFT results for the two $\mu$-oxo complexes, the amount of $4 p$ character that is mixed into the $3 d$ orbitals is given in Table 3 . The total amount of $4 p$ character is less than what is found experimentally, but similar to the experiment, there is only a limited amount of $4 p$ character in the $x z / y z$ orbitals, while more is present in the $z^{2}$ orbital for both $\mu$-oxo complexes. Note that a small amount of $4 p$ character is also found in $\mathrm{Fe}_{2}-\mathrm{OH}-\left(\mathrm{HBpz}_{3}\right)_{2}(\mu-\mathrm{ac})_{2}$ due to the lower than $O_{h}$ symmetry of the actual $\mathrm{Fe}(\mathrm{III})$ site, which leads to an increased intensity at $7114.8 \mathrm{eV}$ in the experimental spectrum and a deviation from the nominal $3 / 2$ ratio of the $t_{2 g} / e_{g}$ intensities (Table 2 ). However, the effect 
is small $(<0.7 \%$ (see Ref. 8)) and does not significantly alter the estimate of $4 p$ character mixed into the $3 d$ orbitals in the oxo-bridged complexes.
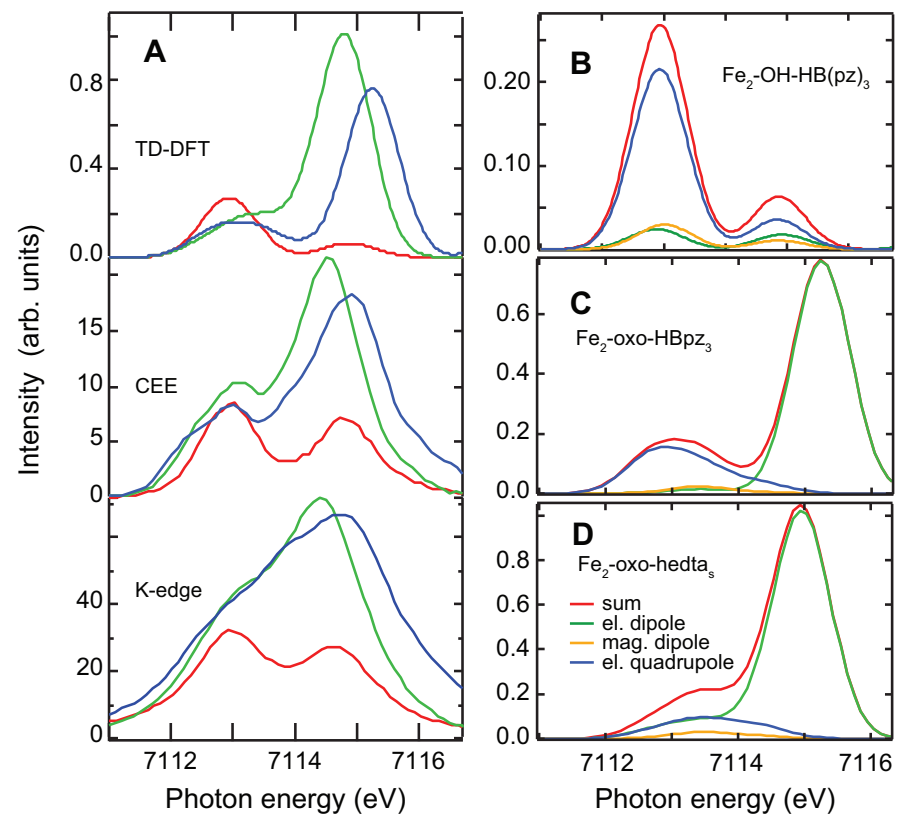

Figure 4: A: Comparison of the TD-DFT derived K pre-edge results (top) with the CEE cuts (middle) and the experimental $\mathrm{K}$ pre-edge (bottom) for $\mathrm{Fe}_{2}-\mathrm{OH}-\left(\mathrm{HBpz}_{3}\right)_{2}(\mu \text {-ac) })_{2}$ (red), $\mathrm{Fe}_{2}$-oxo- $\left(\mathrm{HBpz}_{3}\right)_{2}(\mu \text {-ac) })_{2}$ (blue) and $\mathrm{Fe}_{2}$-oxo-hedta 2 (green). The individual contributions from the electric dipole and quadrupole (green and blue, respectively) together with the magnetic dipole (orange) are shown in B-D for the three complexes. The sum of all three contributions is shown in red.

While the VBCI multiplet analysis is required for fitting the L-edge XAS spectrum, the $\mathrm{K}$ pre-edge can be correlated to TD-DFT calculations. Figure 4A shows the comparison of the experimental K-edges with the TD-DFT derived spectra. The top panel shows the TD-DFT spectra of $\mathrm{Fe}_{2}-\mathrm{OH}-\left(\mathrm{HBpz}_{3}\right)_{2}(\mu \text {-ac })_{2}$ (red), $\mathrm{Fe}_{2}$-oxo- $\left(\mathrm{HBpz}_{3}\right)_{2}(\mu \text {-ac })_{2}$ (blue) and $\mathrm{Fe}_{2}$-oxo-hedta 2 (green), which are compared to the experimental CEE cuts (middle panel) and the K-pre-edge spectra (bottom panel). The three TD-DFT spectra exhibit comparable intensities in the lower energy peak at around $7113 \mathrm{eV}$. At higher energies $(\sim 7115 \mathrm{eV})$ the two oxo-bridged complexes gain significant intensity with the main peak of $\mathrm{Fe}_{2}$-oxo- $\left(\mathrm{HBpz}_{3}\right)_{2}(\mu-$ ac) $_{2}$ (blue) being $0.5 \mathrm{eV}$ higher in energy than $\mathrm{Fe}_{2}$-oxo-hedta 2 (green), consistent with the experimental results. Each calculated spectrum consists of three contributions: electric dipole, magnetic dipole, and electric quadrupole contributions. The TD-DFT calculations 
do not do particularly well with respect to the quadrupole distribution, but nicely reproduce the large intensity increase observed in the oxo bridged dimers due to the dipole contribution. The individual contributions for the three complexes are shown in Figure 4B-D. For $\mathrm{Fe}_{2}-\mathrm{OH}-$ $\left(\mathrm{HBpz}_{3}\right)_{2}(\mu-\mathrm{ac})_{2}$ (B), the spectrum is dominated by the quadrupole contribution (blue) as expected for a high spin ferric complex with quasi $O_{h}$ local symmetry. The calculated spectra for both oxo-bridged complexes $\mathrm{Fe}_{2}$-oxo- $\left(\mathrm{HBpz}_{3}\right)_{2}(\mu \text {-ac })_{2} \quad$ (C) and $\mathrm{Fe}_{2}$-oxo-hedta 2 (D) exhibit a strong electric dipole intensity contribution (green) only at the higher energy side of the spectra, consistent with experiment. The intense electric dipole transition at $\sim 7115 \mathrm{eV}$ derives from excitations into the $z^{2}$ orbital. As observed experimentally above, there is no significant electric dipole intensity contribution from excitations into the $d(x z / y z)$ orbitals, which are also allowed to mix with $4 p_{x, y}$ by group theory.

Extended Pre-Edge Region In the experimental K-edge XAS spectra at 3-4 eV above the pre-edge features, additional intensity is present at around $7118.5 \mathrm{eV}$ in the Fe(III)-oxo dimers. In Figure 5(A-C) this is shown for the background subtracted $1 s 2 p$ RIXS integrated pre-edges (blue) as well as for the CEE cuts (red). A blowup of the extended energy region as well as the non-background subtracted spectra are shown in the Supporting Information. For $\mathrm{Fe}_{2}-\mathrm{OH}-\left(\mathrm{HBpz}_{3}\right)_{2}(\mu-\mathrm{ac})_{2}(\mathrm{~A})$, there is no detectable intensity at around $7118.5 \mathrm{eV}$, while intensity in this region is clearly visible for $\mathrm{Fe}_{2}$-Oxo- $\left(\mathrm{HBpz}_{3}\right)_{2}(\mu-\mathrm{ac})_{2} \quad(\mathrm{~B})$ and also present but weaker for $\mathrm{Fe}_{2}$-oxo-hedta $2(\mathrm{C})$.

Previously, intensity in this energy region has been observed for a number of complexes including ferric- and ferrous-hexacyanide ${ }^{6}$ and Fe(III)-oxalate. However, in these complexes, this additional feature arises from Fe $4 p$ mixing into the unoccupied ligand $\pi^{*}$ orbitals with $t_{1 u}$ symmetry (in $\left.O_{h}\right) \cdot{ }^{6}$ Complexes without unoccupied ligand $\pi^{*}$ orbitals relatively close in energy to the $d$ manifold such as ferric- and ferrous- $\left(\operatorname{tacn}_{2}\right)^{\mathrm{i}}$ do not show a feature in this energy range. ${ }^{6}$ Due to the donating nature of the ligands, this mechanism is also not active for the Fe-oxo/OH complexes in this study.

\footnotetext{
${ }^{\mathrm{i}} \operatorname{tacn}=1,4,7$ triazacyclononane.
} 


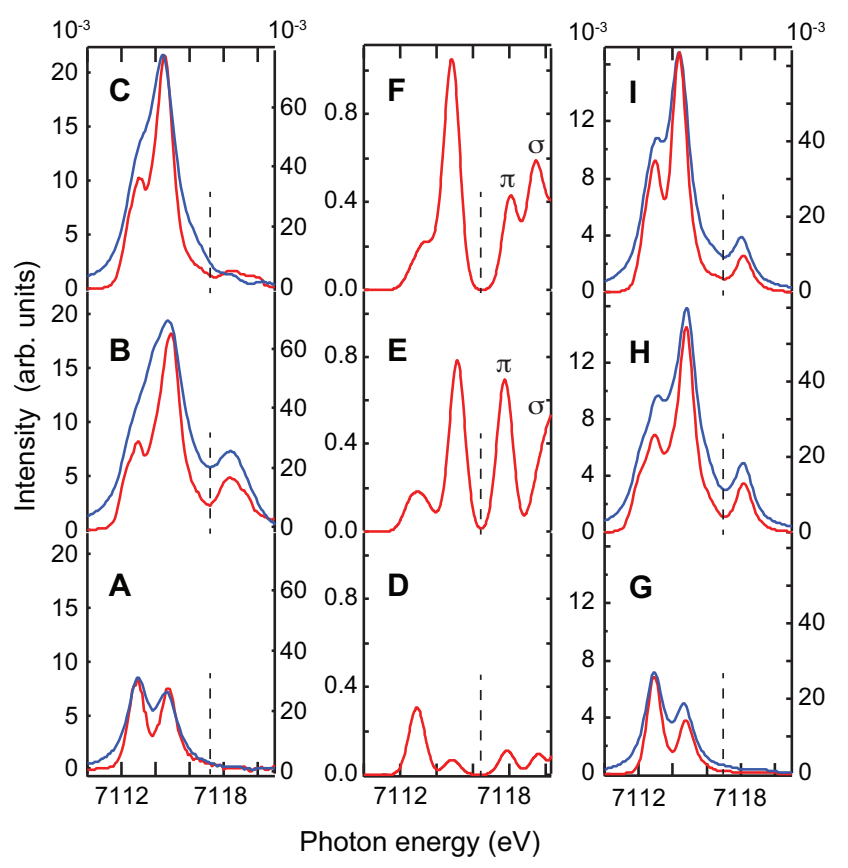

Figure 5: Background subtracted K-edge (blue) and CEE cuts (red) for (A) $\mathrm{Fe}_{2}-\mathrm{OH}$ $\left(\mathrm{HBpz}_{3}\right)_{2}(\mu-\mathrm{ac})_{2}$, (B) $\mathrm{Fe}_{2}$-oxo- $\left(\mathrm{HBpz}_{3}\right)_{2}(\mu \text {-ac })_{2}$, and (C) $\mathrm{Fe}_{2}$-oxo-hedta 2 . The corresponding TD-DFT calculations (K-edge with $1 \mathrm{eV}$ braodening) are shown in D-F and VBCI simulations in G-I. The dashed vertical lines represent the energy separation of the pre-edge (left of line) and extended pre-edge (right of line).

In periodic materials, intensity has been observed from core transitions into non-local states through $\mathrm{Fe}^{(1)}(4 p)-\mathrm{O}(2 p)-\mathrm{Fe}^{(2)}(3 d)$ mixing. ${ }^{10,11,58-61} \mathrm{~A} 1 s$ electron is excited into the $4 p$ character on $\mathrm{Fe}^{(1)}$ that is mixed into the $3 d$ orbitals of the (non-excited) $\mathrm{Fe}^{(2)}$ via an intermediate O $2 p$ orbital (Figure 6 A,B). The resulting intensity appears a few eV higher in energy than the pre-edge, as the electronic relaxation associated with the $1 s$ core-hole creation on $\mathrm{Fe}^{(1)}$ acts on the $d$ valence orbitals of $\mathrm{Fe}^{(1)}$ but not on the $\mathrm{Fe}^{(2)} d$ orbitals. The core-hole on $\mathrm{Fe}^{(1)}$ shifts its $4 p$ down in energy, which allows an increased mixing with the $\mathrm{Fe}^{(2)}(3 d)$ orbitals. This mixing is sensitive to the Fe-O bond length and in particular Fe-O-Fe angle. For the lower energy $3 d(\pi)$ orbitals on $\mathrm{Fe}^{(2)}$, optimal mixing is expected in a 90 degree Fe-O-Fe arrangement due to the $\mathrm{Fe}^{(1)}\left(4 p_{z}\right)-\mathrm{O}\left(2 p_{z}\right) \sigma / \pi$ mixing (Figure $6 \mathrm{~A}$ ), which is more effective than the $\mathrm{Fe}^{(1)}\left(4 p_{x}\right)-\mathrm{O}\left(2 p_{x}\right) \pi / \pi$ mixing that is required for a 180 degree Fe-O-Fe arrangement (Figure 6B). For the $\mathrm{Fe}^{(2)} 3 \mathrm{~d}\left(z^{2}\right)$ delocalized transitions, a high intensity feature in the extended pre-edge region is expected for a linear Fe-O-Fe arrangement. However, this 
$d \sigma$ transition is at higher energy $(\sim 10 \mathrm{Dq})$ and obscured by the rising edge intensity.

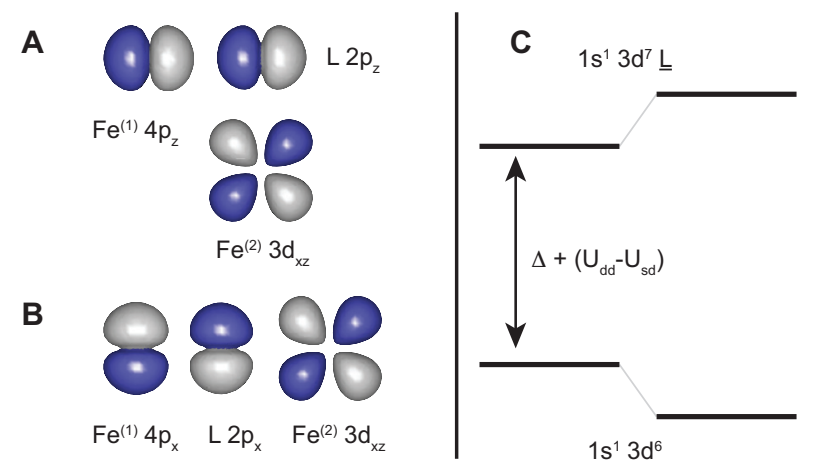

Figure 6: Non-local Fe $4 p-\mathrm{O} 2 p$ - Fe $3 d$ sketch of the orbital interaction for a Fe-O-Fe arrangement of $90^{\circ}(\mathrm{A})$ and $180^{\circ}(\mathrm{B})$. Local LMCT mechanism (C): Energy separation of the LMCT satellite for the final state multi-electron configurations taking charge transfer and Coulomb repulsion energies into account (left) and adding the LMCT mixing (right), which further splits the energy levels. Its intensity gets enhanced through $4 p$ character mixed into the $3 d$ orbitals.

This predicted behavior is reproduced in the TD-DFT calculations of the K-pre-edge (Figure 5D-F). The first higher energy feature at $\sim 7118 \mathrm{eV}$ originates from an $\mathrm{Fe}^{1}(1 s)$ transition into the $\mathrm{Fe}^{(1)}(4 p)-\mathrm{O}(2 p)-\mathrm{Fe}^{(2)}(3 d(x z / y z))$ orbitals. This peak is very weak in $\mathrm{Fe}_{2}$-OH- $\left(\mathrm{HBpz}_{3}\right)_{2}(\mu \text {-ac })_{2}$, has more intensity in $\mathrm{Fe}_{2}$-oxo-hedta 2 and is strongest for $\mathrm{Fe}_{2}$-oxo$\left(\mathrm{HBpz}_{3}\right)_{2}(\mu-\mathrm{ac})_{2}$. While the calculated intensity is higher than the experimentally observed intensity and the energy separation from the pre-edge is lower (3.7 and 4.2 (exp) vs 2.6 and $3.3 \mathrm{eV}$ (TD-DFT) for $\mathrm{Fe}_{2}$-oxo- $\left(\mathrm{HBPz}_{3}\right)_{2}(\mu \text {-ac })_{2}$ and $\mathrm{Fe}_{2}$-oxo-hedta 2 , respectively), the expected angular dependence of the intensity (Figure 6A and $\mathrm{B}$ ) is reproduced with $\mathrm{Fe}_{2}$-oxo$\left(\mathrm{HBpz}_{3}\right)_{2}(\mu \text {-ac })_{2} \quad(\mathrm{Fe}-\mathrm{O}-\mathrm{Fe} \varangle \sim 123)$ being more intense than $\mathrm{Fe}_{2}$-oxo-hedta $2(\varangle \sim 165)$. The $\mathrm{Fe}^{1}(1 s)$ to $\mathrm{Fe}^{(1)}(4 p)-\mathrm{O}(2 p)-\mathrm{Fe}^{(2)}\left(3 d\left(z^{2}\right)\right)$ final state configurations are also calculated to be present at $\sim 1.5-2.5 \mathrm{eV}$ higher in energy relative to the $\mathrm{Fe}^{2} d \pi$ delocalized excitations and also show the expected opposite angular dependence. Core excitation into the $4 s$ orbitals mixed with $4 p^{62}$ appear $\sim 3 \mathrm{eV}$ higher in energy and are buried under the rising edge intensity.

The presence of additional peaks in the extended pre-edge region of the non-centrosymmetric $\mu$-oxo complexes (at $\sim 7118 \mathrm{eV}$ ) is also consistent with the predictions of VBCI simulations for these complexes (Figure 5(G-I)). These features are LMCT satellites that are associated 
with the main pre-edge features. In the multi-electron configuration description, the $3 d^{6} \underline{L}$ configuration is separated in energy from the $3 d^{5}$ configuration by the charge transfer energy $\Delta$ and the LMCT mixing strength, where $\underline{L}$ denotes a ligand hole. In the presence of a $1 s$ core-hole, this energy difference gets corrected by the Coulomb repulsion energy $\Delta+U_{d d}-U_{s d}$, where $U_{d d}$ describes the $3 d-3 d$ and $U_{s d}$ the $1 s-3 d$ Coulomb repulsion. This is shown in the level diagram in Figure 6 C. Its transition energy appears $\sim 3-4 \mathrm{eV}$ above the main pre-edge peak, in the energy region of the additional features in the experimental spectra (Figure $5(\mathrm{~A}-\mathrm{C})$ at $\sim 7118 \mathrm{eV}$ ). The LMCT satellite intensity also gets enhanced through mixing of Fe $4 p$ character into the ligand, which can further mix with the Fe $3 d$ orbitals in the absence of an inversion center. Its intensity therefore depends on $\Delta$ and the LMCT mixing strength, however, it is less pronounced than what is experimentally observed.

Thus, the higher energy features that are observed in the CEE cuts and (less pronounced) in the K-pre-edge of these non-centrosymmetric Fe(III) dimer complexes, have two contributions: the dominant delocalized transition to the neighboring Fe with a strong Fe-O-Fe angle dependence and the weaker LMCT satellite. While both mechanisms lead to a single excitation feature, the experimental feature in the expanded pre-edge region of the CEE cuts is broad and with some structure ( $\sim 1.7 \mathrm{eV}$, see Figure 5(A-C), and expanded in Supporting Information). The experimental data therefore point to contributions from both intensity mechanisms for the higher energy features. Note that the LMCT satellites only have observable intensity in non-centrosymmetric complexes and the non-local transitions only occur in bridged dimers with a highly covalent bridging ligand.

The presence of the LMCT satellite intensity through $4 p$ mixing also has implications for the $1 s 3 d$ and $2 p 3 d$ Coulomb and exchange interaction, $U_{1 s 3 d}$ and $U_{2 p 3 d}$, respectively. Experimentally in $1 s 2 p$ RIXS, the additional intensity in the extended pre-edge region is found on the diagonal through the main peak (Figure 3). In the charge transfer multiplet simulations, this requires $U_{1 s 3 d}=U_{2 p 3 d}+1 \mathrm{eV}$, i.e. more local screening of the $2 p$ core hole. While a difference between $U_{1 s 3 d}$ and $U_{2 p 3 d}$ has only a minor effect on the K-pre-edge region, 
it has a strong effect on the LMCT satellite region (see Supporting Material). Thus $3 d / 4 p$ mixing in non-centrosymmetric sites gives LMCT satellite intensity, enabling $1 s 2 p$ RIXS to be unique in sensitivity to the relative values of $U_{1 s 3 d}$ and $U_{2 p 3 d}$.

\section{L-edge-like CIE cuts}

The advantage of recording $1 s 2 p$ RIXS maps compared to K-edge XAS spectra, is their two-dimensionality. As discussed above, a CEE (diagonal) cut gives a high resolution Kedge XAS-like spectrum, while a CIE (vertical) cut gives an L-edge-like spectrum. ${ }^{5-7,63}$ This has advantages over the direct L-edge XAS in that, by appropriately choosing the incident energy, a transition from the $1 s$ orbital into a selected $d \pi$ or $d \sigma$ orbital can specifically enhance L-edge final states containing this $d \pi$ or $d \sigma$ contribution. The $d \pi$ and $d \sigma$ character in direct L-edge XAS is complicated by multiplet effects from the strong $2 p 3 d$ Coulomb interaction. Alternatively, the direct interpretation of $\pi$ and $\sigma$ character from each CIE cut can be complicated by intensity bleeding effects from peaks at higher incoming and transfer energy due to the large lifetime broadening in the incoming photon energy axis. Figure 7 shows selected CIE cuts for all three complexes (red and green for the OH-bridged complex, and orange, red, green, blue for the oxo-bridged complexes through selected contributions to the K-pre-edge) and compares these to their direct L-edge XAS spectra (shown in blue). Only the $L_{3}$-edge-like region is shown for clarity. The full spectra are given in the Supporting Material.

For $\mathrm{Fe}_{2}-\mathrm{OH}-\left(\mathrm{HBpz}_{3}\right)_{2}(\mu \text {-ac) })_{2}$ (Figure $\left.7 \mathrm{~A}\right)$, the CIE cut through the $d \pi$ region (red curve) leads to an L-edge-like spectrum with a peak at the same energy as the low energy peak of the direct L-edge spectrum (blue). Similarly, the $d \sigma$ CIE cut (green curve) gives an Ledge-like spectrum with a peak corresponding to the main peak of the direct L-edge XAS. Both cuts also show intensity at higher and lower energies, respectively. These point to a small amount of $\sigma$ character in the lower energy L-edge peak, and $\pi$ character in the higher energy L-edge peak, similar to what has been found previously from theoretical simulations 

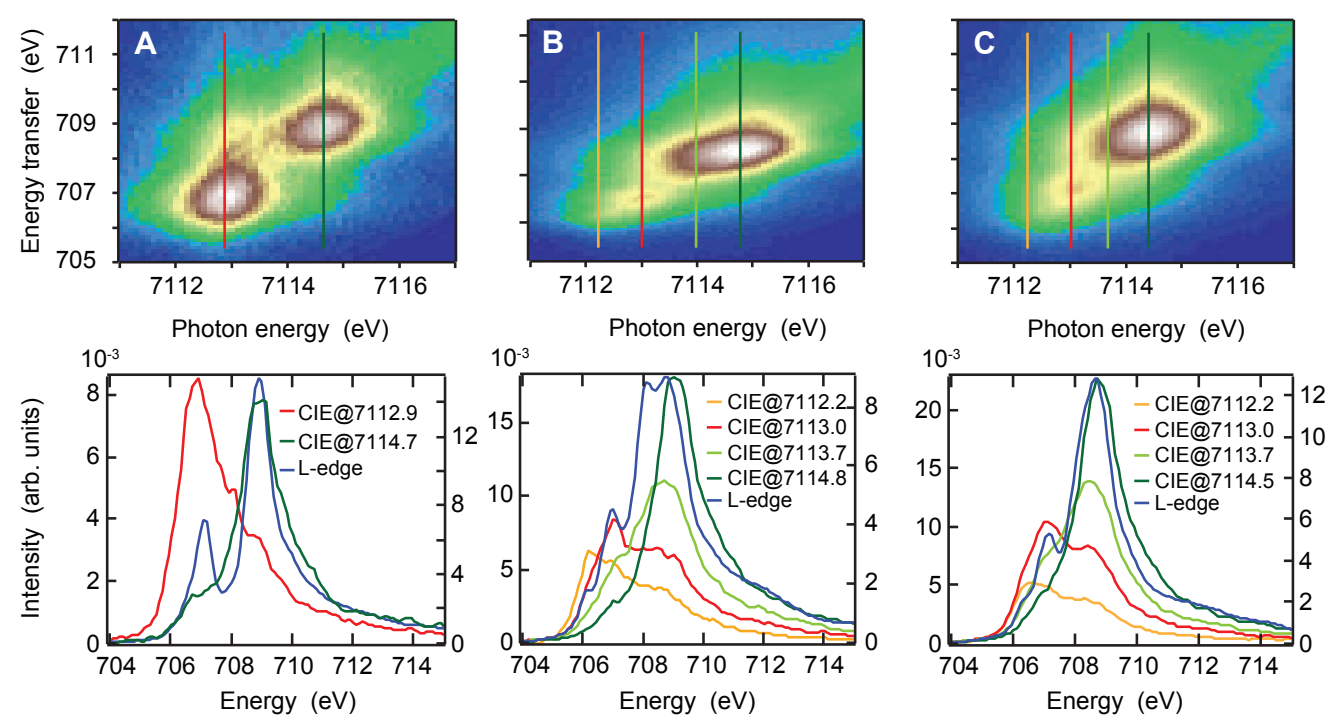

Figure 7: Blow-up of the $\mathrm{L}_{3}$ region of the $1 s 2 p$ RIXS planes together with the CIE cuts at selected energies (vertical lines in RIXS planes) compared to the direct L-edge XAS spectrum: (A) $\mathrm{Fe}_{2}$-OH- $\left(\mathrm{HBpz}_{3}\right)_{2}(\mu \text {-ac })_{2}$, (B) $\mathrm{Fe}_{2}$-oxo- $\left(\mathrm{HBpz}_{3}\right)_{2}(\mu \text {-ac })_{2}$, and (C) $\mathrm{Fe}_{2}$-oxo-hedta 2 .

of L-edge XAS character in $O_{h}$ high spin $\mathrm{Fe}^{I I I}$ complexes. ${ }^{49}$ Due to the width of the RIXS feature in the incoming photon energy direction, however, some intensity bleeds into the $\sigma$ region (for the $d \pi \mathrm{CIE}$ ) and the $\pi$ region (for the $d \sigma \mathrm{CIE}$ ).

For the two oxo-bridged dimer complexes, four CIE cuts at peak energies found from the K-edge and CEE cut fits, are shown together with the direct L-edge XAS. In the oxo-dimer $\mathrm{Fe}_{2}$-Oxo- $\left(\mathrm{HBpz}_{3}\right)_{2}(\mu \text {-ac) })_{2}$ complex (B), the two lower energy CIE cuts (orange and red) show an enhanced splitting in line with a low energy shoulder in the direct L-edge XAS (blue). This indicates the $C_{4 v}$ splitting of the $d \pi$ orbitals. Also the two higher energy CIE cuts at 7113.7 (light green) and $7114.8 \mathrm{eV}$ (dark green) at the $1 s \rightarrow 3 d\left(x^{2}-y^{2}\right)$ and $1 s \rightarrow 3 d\left(z^{2}\right)$ transition energies, respectively, show a splitting of $0.4 \mathrm{eV}$. This splitting is also visible in the direct L-edge XAS spectrum, where two intense peaks with an energy separation of $0.6 \mathrm{eV}$ are observed. The $3 d\left(z^{2}\right)$ intensity is electric dipole resonance enhanced (from the $4 p_{z}$ mixed into the $3 d_{z^{2}}$ ) and appears on the higher energy side of the L-edge XAS spectrum.

For $\mathrm{Fe}_{2}$-oxo-hedta 2 , a CIE cut through the lower energy side of the low energy $1 s 2 p$ RIXS feature (orange) has its maximum aligned with a weak low energy shoulder in the direct L-edge XAS spectrum (blue) at $706.4 \mathrm{eV}$. The CIE cut across the maximum of the 
$d \pi$ feature (red) has its maximum aligned with the maximum of the low energy feature of the direct L-edge XAS spectrum. The L-edge XAS low energy feature therefore contains a large amount of $d \pi$ character and the small shoulder in the L-edge $d \pi$ region originates from a splitting of the $d \pi$ orbitals again due to the $\mathrm{C}_{4 \mathrm{v}}$ symmetry. Additional intensity is also present in the higher energy part of the CIE cut, which mostly originates from a bleed-over from the intensity of the strong feature in the $1 s 2 p$ RIXS plane at higher energy. The two cuts through the $d \sigma$ region (light and dark green) lead to two L-edge-like spectra separated by approximately $0.3 \mathrm{eV}$ with the higher energy cut (dark green) through the $4 p$ dipole intensity enhanced region appearing at the higher energy side of the L-edge spectrum. Since the $4 p$ mixing is into the $d\left(z^{2}\right)$ orbital, we can identify the high energy side of the L-edge XAS spectrum as originating mostly from the $2 p \rightarrow 3 d\left(z^{2}\right)$ transition.
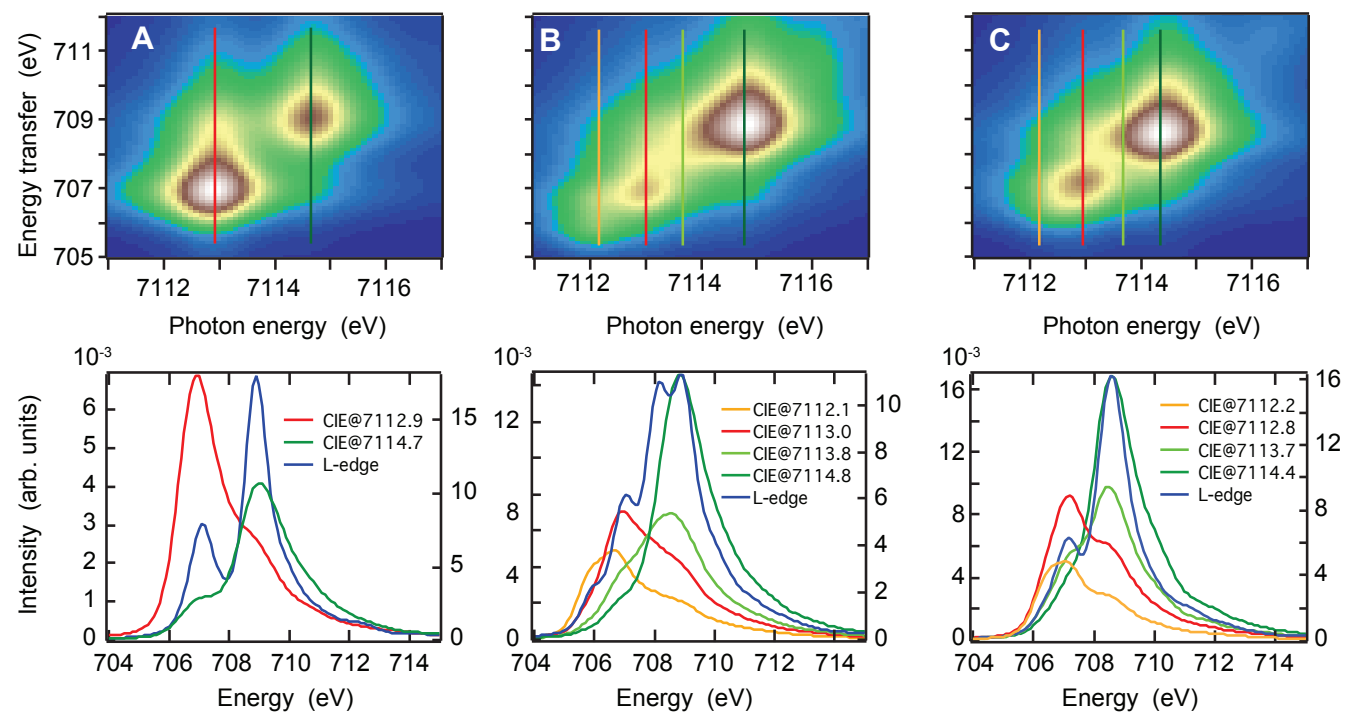

Figure 8: VBCI simulations of the $1 s 2 p$ RIXS planes: Blow-up of the $\mathrm{K} \alpha_{1}\left(L_{3}\right)$ region together with the CIE at selected energies (vertical lines in RIXS map) compared to the direct L-edge XAS simulations: (A) $\mathrm{Fe}_{2}-\mathrm{OH}-\left(\mathrm{HBpz}_{3}\right)_{2}(\mu \text {-ac })_{2},(\mathrm{~B}) \mathrm{Fe}_{2}$-oxo- $\left(\mathrm{HBpz}_{3}\right)_{2}(\mu \text {-ac })_{2}$, and $(\mathrm{C}) \mathrm{Fe}_{2}$-oxo-hedta 2 .

The $1 s 2 p$ RIXS VBCI simulations with the parameters used to derive the DOC in Table 1 successfully reproduce the experimental observations, including all energy splittings and relative intensities as shown in Figure 8. Thus these charge transfer multiplet calculations are capable of properly simulating the electronic structures of non-centrosymmetric complexes 
from direct L-edge XAS and $1 s 2 p$ RIXS spectra. The $2 \mathrm{D}$ nature of the $1 s 2 p$ RIXS maps with the ability to compare individual cuts (CIE and CEE) for different data sets, provides an enhanced sensitivity of the VBCI fits relative to fits only to the L-edge XAS. This is despite strong electric dipole transition contributions from $3 d / 4 p$ mixing that does not significantly contribute to the direct L-edge XAS (see also the Supporting Information). Therefore, combining both methods give the most accurate solution space for the parameters used in the VBCI analysis and thus the DOCs in Table 1.

\section{DOC from $1 s 2 p$ RIXS alone}

In the analysis above, the combination of L-edge XAS and $1 s 2 p$ RIXS data have been used together to simulate spectra and to obtain DOCs. A goal of the present study is to use $1 s 2 p$ RIXS for systems where L-edge XAS is not accessible (as in a metallo-enzyme intermediate in solution) to obtain DOC of the associated Frontier Molecular Orbitals involved in reactivity. Further, this needs to be accomplished for non-centrosymmetric systems as many of the key intermediates are $\mathrm{Fe}^{I V}=\mathrm{O}$ heme enzymes. We therefore constructed an approach to extract DOC from data where only the $1 s 2 p$ RIXS plane is available for the intermediate, but when both L-edge XAS and $1 s 2 p$ RIXS are available on a related model complex. Here, we treat the $\mathrm{Fe}_{2}$-oxo-hedta 2 complex as the "intermediate" where only RIXS data are available and $\mathrm{Fe}_{2}$-oxo- $\left(\mathrm{HBpz}_{3}\right)_{2}(\mu \text {-ac })_{2}$ as the well defined related model complex. For this, we searched for key spectral features in the 2D RIXS maps that allow a step-wise progression between the RIXS planes from $\mathrm{Fe}_{2}$-oxo- $\left(\mathrm{HBpz}_{3}\right)_{2}(\mu \text {-ac })_{2}$ to $\mathrm{Fe}_{2}$-oxo-hedta 2 . Figure 9 shows a collection of experimental $1 s 2 p$ RIXS cuts (solid curves) together with the corresponding simulations (dashed curves) for $\mathrm{Fe}_{2}$-oxo- $\left(\mathrm{HBpz}_{3}\right)_{2}(\mu \text {-ac) })_{2}$ (red) and $\mathrm{Fe}_{2}$-oxo-hedta ${ }_{2}$ (black). Four characteristic changes are observed in going from the reference $\mathrm{Fe}_{2}$-oxo- $\left(\mathrm{HBpz}_{3}\right)_{2}(\mu-$ ac) $)_{2}$ to $\mathrm{Fe}_{2}$-oxo-hedta 2 : (i) The change in the overall spectral shape of the CEE cuts in the pre-edge region (7111-7117 eV, Figure 9A). The energy splitting between the $\sigma$ and $\pi$ regions decreases, together with an increased $\pi$ energy splitting. (ii) The CIE cut through 


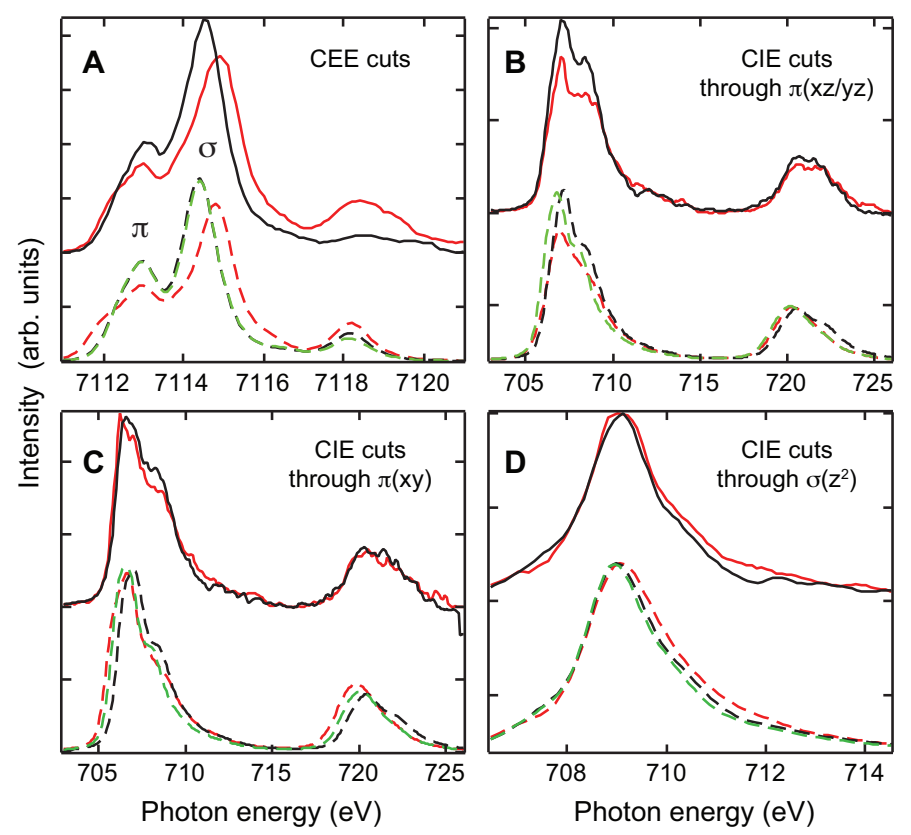

Figure 9: Characteristic spectral changes when going from $\mathrm{Fe}_{2}$-oxo- $\left(\mathrm{HBpz}_{3}\right)_{2}(\mu \text {-ac })_{2}$ to $\mathrm{Fe}_{2^{-}}$ oxo-hedta $_{2}$. A: Comparison of the CEE cuts. B: CIE cuts through the $\pi(x z / y z)$ feature at $7113 \mathrm{eV}$. C: CIE cuts through the lower energy feature of $\pi$ character $(x y)$ at $7112.2 \mathrm{eV} \mathrm{D:}$ CIE cuts through the main intensity dipole region $\left(3 d\left(z^{2}\right)\right.$ symmetry) normalized to one and shifted such that the spectra are energy aligned at half intensity. - Color scale: Red solid - experimental $\mathrm{Fe}_{2}$-oxo- $\left(\mathrm{HBpz}_{3}\right)_{2}(\mu \text {-ac })_{2}$; black solid - experimental $\mathrm{Fe}_{2}$-oxo-hedta 2 ; dashed red - simulated $\mathrm{Fe}_{2}$-oxo- $\left(\mathrm{HBpz}_{3}\right)_{2}(\mu \text {-ac })_{2}$; dashed black - simulated $\mathrm{Fe}_{2}$-oxo-hedta 2 ; dashed green - simulated $\mathrm{Fe}_{2}$-oxo-hedta 2 with RIXS only.

the $d \pi(x y / z y)$ feature of the RIXS plane at $7113.0 \mathrm{eV}$ incident energy (Figure 9A) shows an increase in intensity (Figure 9B) due to a decreased $\pi(x z / y z)$ LMCT mixing. (iii) $1 s \rightarrow 3 d$ excitation into the $x y$ orbital appears on the low energy side of the $d \pi$ peak at $7112.2 \mathrm{eV}$ in Figure 9A. CIE cuts at this energy show a similar intensity for both Fe-oxo complexes. (Figure 9C) (iv) The CEE pre-edges of the $\mathrm{Fe}_{2}$-oxo dimer complexes at 7114.8 and 7114.4 $\mathrm{eV}$ in Figure $9 \mathrm{~A}$ are dominated by electric dipole transitions due to $4 p_{z}\left(a_{1}\right)$ character mixed in the $3 d\left(z^{2}\right)$ orbitals. The CIE cuts through these energies (Figure 9D) show a narrower $\mathrm{L}_{3}$-edge-like peak for $\mathrm{Fe}_{2}$-oxo-hedta ${ }_{2}$.

Based only on these observed spectral changes starting from the well-defined $\mathrm{Fe}_{2}$-oxo$\left(\mathrm{HBpz}_{3}\right)_{2}(\mu \text {-ac })_{2}$ complex (from L-edge XAS and $1 s 2 p$ RIXS), the $1 s 2 p$ RIXS only $\mathrm{Fe}_{2}$-oxohedta $_{2}$ plane could be simulated (green dashed line in Figure 9). The corresponding DOC 
Table 4: DOC values derived from the full data set (L-edge $+1 s 2 p$ RIXS) for $\mathrm{Fe}_{2}$-oxo$\left(\mathrm{HBpz}_{3}\right)_{2}(\mu \text {-ac })_{2}$ and $\mathrm{Fe}_{2}$-oxo-hedta ${ }_{2}$, as well as for using the $\mathrm{Fe}_{2}$-oxo-hedta 2 1s2p RIXS plane alone. Note that the first two rows are a repetition from Table 1 for simplicity. Note that the value for $\mathrm{Fe}(e)$ is per orbital and the $e$ set carries twice the amount.

\begin{tabular}{|c|c|c|c|c|c|c|}
\hline & \multicolumn{5}{|c|}{ DOC metal character (\%) } \\
\hline & & $\mathrm{Fe}\left(b_{2}\right)$ & $\mathrm{Fe}(e)$ & $\mathrm{Fe}\left(b_{1}\right)$ & $\mathrm{Fe}\left(a_{1}\right)$ & total \\
\hline $\mathrm{Fe}_{2}-o x o-\left(H B p z_{3}\right)_{2}(\mu-a c)_{2}$ & $(L-e d g e+R I X S)$ & 89 & 80 & 77 & 67 & 392 \\
\hline $\mathrm{Fe}_{2}$-oxo-hedta $a_{2}$ & $(L-e d g e+R I X S)$ & 91 & 84 & $7 y$ & 69 & 405 \\
\hline $\mathrm{Fe}_{2}$-oxo-hedta 2 & (RIXS only) & 90 & 85 & 79 & 71 & 410 \\
\hline
\end{tabular}

values are given in Table 4 bottom row, together with the L-edge + RIXS derived values for $\mathrm{Fe}_{2}$-oxo- $\left(\mathrm{HBpz}_{3}\right)_{2}(\mu \text {-ac })_{2}$ and $\mathrm{Fe}_{2}$-oxo-hedta 2 taken from Table 1 (in italic). As also found from the full data set simulations in Table 1, RIXS only simulations find $\mathrm{Fe}_{2}$-oxohedta 2 less covalent than $\mathrm{Fe}_{2}$-oxo- $\left(\mathrm{HBPz}_{3}\right)_{2}(\mu \text {-ac })_{2}$, with larger increases in $d(x z / y z) \pi$ and $d\left(z^{2}\right) \sigma$ character. Thus a large number of characteristic quantitative differences (relative to a well defined model) are available to properly simulate the $1 s 2 p$ RIXS plane in the absence of an L-edge XAS spectrum, despite the strong spectral distortion associated with the electric dipole intensity contributions from $3 d / 4 p$ mixing in non-centrosymmetric complexes.

\section{Discussion}

For systems containing local inversion symmetry, only electric quadrupole allowed $1 s \rightarrow 3 d$ transitions occur, allowing the direct extraction of the DOC from $1 s 2 p$ RIXS with the advantage of accessing L-edge XAS forbidden final states. ${ }^{6}$ Upon loss of inversion symmetry, $4 p$ character can mix into the $3 d$ orbitals, giving an electric dipole allowed $1 s \rightarrow 4 p$ intensity contribution to the K-pre-edge region and therefore significantly enhancing their intensity. ${ }^{8,10-12,56,57}$

In going from $\mathrm{Fe}_{2}-\mathrm{OH}-\left(\mathrm{HBpz}_{3}\right)_{2}(\mu \text {-ac })_{2}$ to $\mathrm{Fe}_{2}$-oxo- $\left(\mathrm{HBpz}_{3}\right)_{2}(\mu \text {-ac })_{2}$, the Fe-O bridge bond length shortens leading to a loss of local inversion symmetry to $C_{4 v}$ enabling $4 p$ mixing into specific $3 d$ orbitals, and a splitting of the $\pi$ and $\sigma$ orbitals that is visible in the L- 

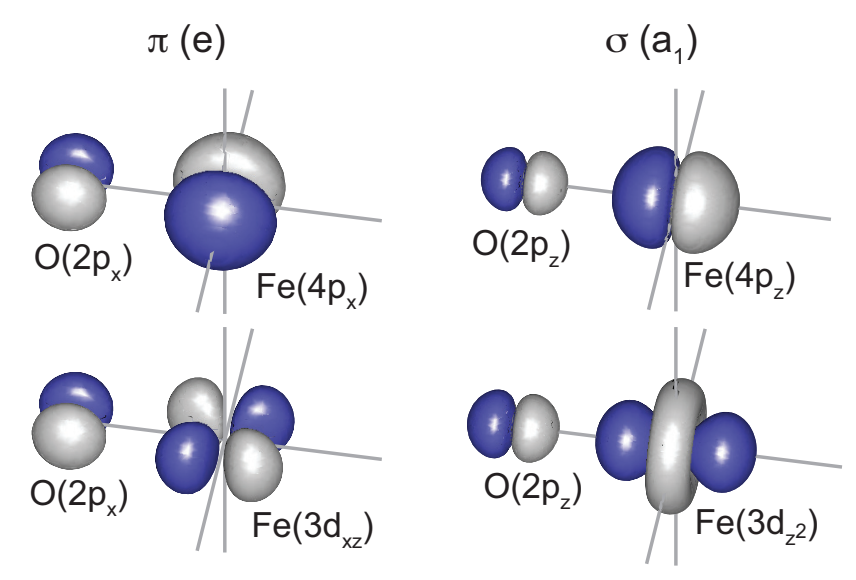

Figure 10: Sketch of the orbitals involved in $e$ (left) and $a_{1}$ (right) $3 d / 4 p$ mixing via ligand mixing for the ligand $2 p$ - metal $3 d$ (bottom) and ligand $2 p$ - metal $4 p$ orbitals (top).

edge XAS, $1 s 2 p$ RIXS and CEE spectra (Figures 2 and 3). Simultaneous L-edge XAS and $1 s 2 p$ RIXS multiplet simulations that now include the $4 p$ mixing were developed and used to determine the DOC (Table 1). For $\mathrm{Fe}_{2}$-oxo- $\left(\mathrm{HBPz}_{3}\right)_{2}(\mu \text {-ac })_{2}$ they give increased oxo covalent mixing into the $d \pi$ and $d \sigma$ orbitals, which are the frontier molecular orbitals (FMOs) in metal-oxo systems, that activate reactivity perpendicular to and along the $\mathrm{Fe}=\mathrm{O}$ bond, respectively.

While both the $d\left(z^{2}\right)$ and the $d(x z / y z)$ orbitals have high covalencies in the oxo bridged complexes and are allowed to mix with the $4 p$ orbitals $\left(a_{1}\left(p(z) / d\left(z^{2}\right)\right)\right.$ and $\left.e(p(x, y) / d(x z, y z))\right)$, only $4 p(z)$ mixing into the $3 d\left(z^{2}\right)$ orbital is experimentally found to be significant based experiment and TD-DFT calculations. As shown in Ref 10, $p$ mixing into $d$ orbitals originates from their simultaneous overlap with the same ligand valence orbital. This selective $3 d\left(z^{2}\right) / 4 p(z)$ mixing in oxo bridged Fe dimers can therefore be explained by a simple orbital overlap picture. Figure 10 shows a sketch of the orbitals involved in $e$ (left) and $a_{1}$ (right) mixing via interaction with a $p$ ligand valence orbital. The $e$ overlap is of $\pi$ character and this directionality limits the mixing between the oxo $2 p(x / y)$ and the metal $4 p(x / y)$ orbitals. The $a_{1}$ overlap is of $\sigma$ character, leading to strong oxo $2 p_{z}$ and Fe $4 p(z)$ overlap and a dominant contribution of the $3 d\left(z^{2}\right)-4 p(z)$ mixing to the spectral shape, i.e. the observed enhanced intensity of only the $a_{1}$ channel. 
The two dimensionality of the $1 s 2 p$ RIXS maps generally allows excitations of selected K-pre-edge intermediate states and, via vertical CIE cuts, projections of specific final states in the direct L-edge XAS spectrum that are identified through the specific symmetry of the allowed $3 d / 4 p$ mixing. The enhanced intensity of only the $a_{1}$ channel described above additionally enables the selective determination of $d_{z^{2}}$ character in the L-edge XAS spectra of $\mathrm{Fe}_{2}$-oxo- $\left(\mathrm{HBpz}_{3}\right)_{2}(\mu \text {-ac })_{2}$ and $\mathrm{Fe}_{2}$-oxo-hedta ${ }_{2}$ through the strong enhancement of $a_{1}$ states in the L-edge-like CIE cuts.

Correlation of the two oxo-bridged complexes through the results of the DOC from VBCI simulations of the RIXS data and Mulliken analyses of the associated DFT calculations, provides further insight into the differences in their electronic structure. In quantitatively comparing the $\mathrm{HB}(\mathrm{pz})_{3}(\mu-\mathrm{ac})_{2}$ to the hedta ${ }_{2}$ ligand systems the total covalency of the FeO-Fe in the latter is lower both in experiment and DFT calculations, particularly for the $d \pi$ orbitals. Interestingly, both the oxo and total ligand contribution in the ferric unoccupied $d$ orbitals have decreased. As the Fe-O-Fe bond lengths are the same, this decrease in the covalency of the hedta ligated complex can have two possible origins: i) the increase in Fe-O-Fe angle from 125 to 164.9 degrees, or ii) the difference in the ligand systems. Both possibilities are tested in the Supporting Information. Variation in the Fe-O-Fe angle with fixed hedta ligand has no effect. Calculations on hypothetical Fe $\mathrm{F}^{\mathrm{III}}$-oxo monomers with the same ligations show that the hedta is a better donor than the $\left(\mathrm{HBpz}_{3}\right)(\mu$-ac) ligation. In $\mathrm{Fe}^{\mathrm{III}}$-oxo monomers this is compensated by the oxo donation leading to equivalent $d$ character in both. In the oxo bridged dimers the oxo donation is shared between the two $\mathrm{Fe}^{\mathrm{III}}$ leading to less oxo donation per $\mathrm{Fe}$ and in the $\left(\mathrm{HBpz}_{3}\right)_{2}(\mu \text {-ac })_{2}$ complex increased ligand donation to compensate. However, in the $\mathrm{Fe}_{2}$-oxo-hedta 2 complex the larger negative charge on each ligand limits its ability to donate and an increased net Fe character is observed both in RIXS data and calculations. In contrast to a lack of Fe-O-Fe angle effect on the covalency of the oxo bond, there is an effect on the extended pre-edge region where the experimental intensity decreases with increasing angle. This delocalized transition is unique to the dimers 
and reflects the oxo $2 p$ superexchange pathway between the two Fe centers. However, in contrast to the $3 d / 4 p$ single site mixing responsible for the dipole intensity of the pre-edge transition that involves the oxo $2 p_{z}$ orbital, the delocalized transition intensity requires an oxo $2 p_{x, y}$ mechanism (for the $90^{\circ}$ angle) as it involves mixing into the low energy $d \pi$ of the adjacent Fe.

By going from a complex with a known structure and using the insight from the spectral features in the 2D $1 s 2 p$ RIXS data to an unknown complex, as we did by going from $\mathrm{Fe}_{2}-\mathrm{OH}-$ $\left(\mathrm{HBpz}_{3}\right)_{2}(\mu \text {-ac })_{2}$ to $\mathrm{Fe}_{2}$-oxo- $\left(\mathrm{HBpz}_{3}\right)_{2}(\mu \text {-ac })_{2}$ and to $\mathrm{Fe}_{2}$-oxo-hedta 2 , a clear assignment of spectral changes in different energy regions and hence elucidating the orbital contributions to these regions becomes possible. These RIXS correlations enable specific assignments of features in their corresponding CEE cuts (also called high energy resolution fluorescence detection (HERFD) spectra). These alone can then be used to assist in assigning HERFD spectra of related complexes. This approach provides insight into the structures of biferric peroxo intermediates $\mathrm{P}^{\prime}$ in 4-aminobenzoate N-oxygenase (AurF) and $\mathrm{P}$ in ribonucleotidereductase (RNR), for which HERFD spectra were recently published by Cutsail et al. ${ }^{62}$ Here, we use the assignments of the 2D $1 s 2 p$ RIXS planes of the two pyrazolyl borate complexes $\mathrm{Fe}_{2}-\mathrm{OH}-\left(\mathrm{HBpz}_{3}\right)_{2}(\mu-\mathrm{ac})_{2}$ and $\mathrm{Fe}_{2}$-Oxo- $\left(\mathrm{HBpz}_{3}\right)_{2}(\mu-\mathrm{ac})_{2}$ to interpret their HERFD spectral features. We then go from these structures with two $\mu$-1,3-carboxylate bridges and a $\mu$ hydroxo or $\mu$-oxo, respectively, to structures with two $\mu$-1,3-carboxylates and a $\mu$-1,2-peroxo or a $\mu$-1,2-hydroperoxo bridge.

Figure 11 A shows the HERFD data for the model complexes $\mathrm{Fe}_{2}-\mathrm{OH}-\left(\mathrm{HBpz}_{3}\right)_{2}(\mu$ ac) $)_{2}$ (blue) and $\mathrm{Fe}_{2}$-oxo- $\left(\mathrm{HBpz}_{3}\right)_{2}(\mu \text {-ac })_{2}$ (green) (i.e. CEE cuts from Figure 3), and the biferric enzyme intermediates $\mathrm{P}^{\prime}$ of $\operatorname{AurF}(\mathrm{red})^{62}$ and $\mathrm{P}$ in RNR (Ec $\beta$-W48F/D84E) (yellow). ${ }^{62}$ Relative to the $\mu$-hydroxo bridged complex (blue), the intensity in the spectrum of $\mathrm{P}$ in RNR (yellow) increases, but not as much as for the $\mu$-oxo bridged model complex (green). The $\mathrm{P}^{\prime}$ in AurF spectrum (red) shows only a slightly higher total intensity than $\mathrm{Fe}_{2}-\mathrm{OH}-$ $\left(\mathrm{HBpz}_{3}\right)_{2}(\mu \text {-ac })_{2}$ with the additional intensity found at $\sim 7113.5 \mathrm{eV}$ leading to a broad peak 

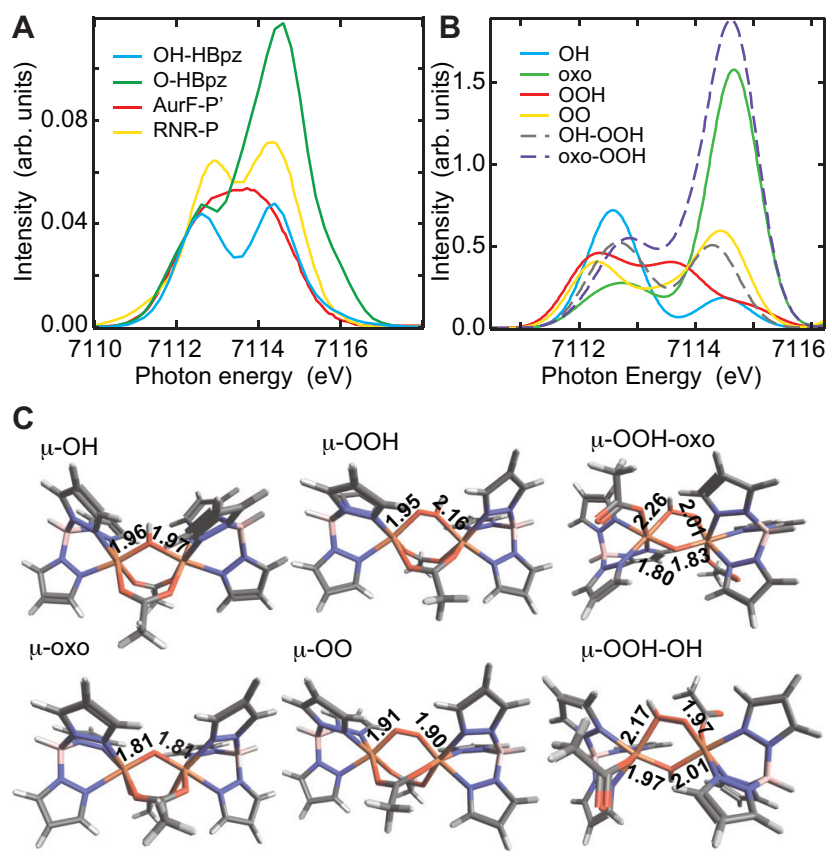

Figure 11: A: Experimental HERFD data for $\mathrm{Fe}_{2}-\mathrm{OH}-\left(\mathrm{HBpz}_{3}\right)_{2}(\mu \text {-ac })_{2}$ (blue), $\mathrm{Fe}_{2}$-oxo$\left(\mathrm{HBpz}_{3}\right)_{2}(\mu \text {-ac })_{2}$ (green), AurF-P' (red) and RNR-P (yellow). The AurF-P' and RNR-P data are adapted from Ref. 62. B: TD-DFT calculations of $\mathrm{Fe}_{2}-\mathrm{X}-\left(\mathrm{HB}(\mathrm{pz})_{3}\right)_{2}(\mu-\mathrm{ac})_{2}$ with $\mathrm{X}=$ oxo (solid green), hydroxo (solid blue), peroxo (solid yellow), hydroperoxo (solid red), as well as of $\mathrm{Fe}_{2}-\mathrm{X}-\mathrm{Y}-\left(\mathrm{HB}(\mathrm{pz})_{3}\right)_{2}(\mathrm{ac})_{2}$ with $\mathrm{X}=$ hydroperoxo and $\mathrm{Y}=$ hydroxo (dashed grey) and oxo (dashed purple), and monodentate carboxylates. C: Their corresponding DFT optimized structures.

with little structure.

For additional insight, we obtained optimized structures for $\mathrm{Fe}_{2}-\mathrm{OH}-\left(\mathrm{HBpz}_{3}\right)_{2}(\mu \text {-ac })_{2}$ and $\mathrm{Fe}_{2}$-oxo- $\left(\mathrm{HBpz}_{3}\right)_{2}(\mu \text {-ac })_{2}$ that agree with crystallography (Figure $11 \mathrm{C}$, left), used these as references for the hypothetical complexes $\mathrm{Fe}_{2}$-hydroperoxo- $\left(\mathrm{HBpz}_{3}\right)_{2}(\mu \text {-ac })_{2}$ and $\mathrm{Fe}_{2}$-peroxo$\left(\mathrm{HBpz}_{3}\right)_{2}(\mu \text {-ac })_{2}$ (middle), where the $\mathrm{P}$ complex in RNR has been shown to be a cis- $\mu-1,2-$ peroxo by resonance Raman ${ }^{64}$ and the $\mathrm{P}^{\prime}$ in AurF a cis- $\mu$-1,2-hydroperoxo from NRVS, ${ }^{20}$ vand calculated their TD-DFT spectra (Figure $11 \mathrm{~B}$ ). The theoretical spectra reasonably correlate to the experimental spectra for the related biferric enzyme peroxo intermediates.

The calculated $\mu$-peroxo complex has Fe-O distances of $1.91 \AA$, which are shorter than the Fe-O bond length of $\mu$-hydroxo $(1.96 \AA)$ but longer than in $\mu$-oxo (1.81 $\AA$ ). Consistent with these bond length trends, the observed moderate increase in the $3 d\left(z^{2}\right)$ intensity at $\sim 7114.6$ 
$\mathrm{eV}$ in the experimental RNR-P spectrum is therefore due to $4 p(z)$ mixing for both Fe relative to the $\mu$-hydroxo model complex, but much less than in the $\mu$-oxo bridged model complex, as it is also observed in their calculated TD-DFT spectra. These results are consistent with resonance Raman results.

In going to $\mathrm{P}^{\prime}$ in AurF the HERFD intensity is lower than in RNR-P. This is consistent with NRVS data on $\mathrm{P}^{\prime}$ in AurF. In our DFT optimized structure of the $\mu$-hydroperoxo complexe, the Fe-O distance for the non-protonated oxygen of the peroxo is the same as in the $\mu$-hydroxo $(1.95 \AA)$ while the $\mathrm{Fe}-\mathrm{OH}$ distance for the protonated oxo is significantly longer $(2.16 \AA)$. This leads to a ligand field (LF) splitting of the $z^{2}$ peaks for each Fe with the transition to the Fe with the protonated oxygen being at lower energy, but gaining intensity through additional $4 p(z)$ mixing. Based on the additional intensity in the HERFD spectrum of $\mathrm{P}^{\prime}$ in AurF at $\sim 7113.5 \mathrm{eV}$, this LF prediction is observed in the experimental spectrum, and confirmed in the TD-DFT calculated spectrum where the $d\left(z^{2}\right)$ from the two $\mathrm{Fe}^{\mathrm{III}}$ splits in energy producing the new feature at $\sim 7113.5 \mathrm{eV}$. Recent resonance Raman data on $\mathrm{P}^{\prime}$ in CmlI, an intermediate equivalent to $\mathrm{P}^{\prime}$ in AurF, have suggested that there may also be a $\mu$-oxo bridge present in this intermediate. ${ }^{65}$ This would have a strong impact on its reaction coordinate as an oxo bridge would stabilize the biferric site against the single electron transfer computationally derived from $\mathrm{P}^{\prime}$ in AurF. The HERFD data in Figure 11 A for $\mathrm{P}^{\prime}$ in AurF refute the presence of an additional $\mu$-oxo bridge and are alternatively consistent with a $\mu$-hydroxo bridge in addition to the $\mu$-1,2-hydroperoxo. Inclusion of an additional $\mu$-oxo (dashed purple in Figure $11 \mathrm{~B}$ ) into the $\mu$-1,2-hydroperoxo structure leads to an intensity increase at $7114.8 \mathrm{eV}$ that is not observed experimentally. Inclusion of an additional $\mu$-hydroxo (dashed grey) leads to a two feature spectrum without the additional intensity at $\sim 7113.5 \mathrm{eV}$.

While the above analysis of the $1 s 2 p$ RIXS planes and L-edge XAS spectra of structurally aligned models allowed for a clear interpretation of the HERFD data for the corresponding peroxo intermediates, the availability of full $1 s 2 p$ RIXS planes on these would have been 
preferred as we would have then been able to extract the DOC and therefore obtain insight into the FMOs. While for $\mathrm{P}$ in RNR the two Fe sites are close to equivalent, this is not the case for $\mathrm{P}^{\prime}$ in AurF due to protonation of the peroxo. This leads to the two peaks corresponding to the $z^{2}$ transitions on each Fe in the HERFD spectra. This splitting would allow tuning into the $z^{2}$ of each Fe ion by individual vertical (i.e. CIE) cuts through the RIXS plane to enhance the L-edge-like spectrum and hence the ligand field information that is associated with each Fe.

\section{Conclusion}

The $1 s 2 p$ RIXS intensity of non-centrosymmetric metal systems is strongly affected by electric dipole character resulting from metal $4 p$ mixing into the $3 d$ orbitals. This mixing reflects the frontier molecular orbitals that are key to reactivity in metal-oxo intermediates. This strongly affects the CEE cuts and results in the $3 d / 4 p$ selective enhancement of specific L-edge final states in the CIE cuts.

A methodology is developed to include the $\mathrm{p} / \mathrm{d}$ mixing via covalent overlap with the ligand in the multiplet analysis of $1 s 2 p$ RIXS planes and to extract DOC information from enzyme intermediates in solution, providing $1 s 2 p$ RIXS data are available on relevant models. In the present study bridged biferric model RIXS data were extended to obtain structural insight into biferric non-heme peroxo enzyme intermediates and in ongoing studies will be applied on $\mathrm{Fe}(\mathrm{IV})=\mathrm{O}$ intermediates as their oxo-Fe(IV) bond strongly affects their spectral shape in regions important to $\pi$ and $\sigma$ FMO contributions to reactivity. 


\section{Supporting Information Available}

The Supporting Information is available free of charge on the ACS Publications website. Additional information and details are given on the experimental methods (L-edge and $1 s 2 p$ RIXS), $3 d 4 p$ charge transfer multiplet simulations including a detailed description of the model, absolute intensities and used parameters, as well as details on the data fitting routines and results, additional information, details and explanations on the parameter dependence of the satellite energy position, and the full CIE/L-edge cut region is shown. Hypothetical monomer Fe-oxo model complexes are described for comparison with the Fe-oxo dimers complexes.

\section{Acknowledgement}

This work was supported by the National Institutes of Health (Grant GM-40392 to E.I.S.). Use of the Stanford Synchrotron Radiation Lightsource (SSRL), SLAC National Accelerator Laboratory, is supported by the US Department of Energy (DOE), Office of Science, Office of Basic Energy Sciences under Contract DE-AC02-76SF00515. The SSRL Structural Molecular Biology Program is supported by the DOE Office of Biological and Environmental Research, and by the National Institutes of Health, National Institute of General Medical Sciences (Grant P41GM103393 to K.O.H and B.H). T.K. acknowledges financial support by the German Research Foundation (DFG) Grant KR3611/2-1. M.L.B. acknowledges the support of the Human Frontier Science Program and The Royal Society of Chemistry (RM1802-4019). M.L. acknowledges support from the Marcus and Amalia Wallenberg Foundation. 


\section{References}

(1) Baker, M. L.; Mara, M. W.; Yan, J. J.; Hodgson, K. O.; Hedman, B.; Solomon, E. I. Coordination Chemistry Reviews 2017, 345, 182-208.

(2) Wasinger, E. C.; de Groot, F. M. F.; Hedman, B.; Hodgson, K. O.; Solomon, E. I. Journal of the American Chemical Society 2003, 125, 12894-12906.

(3) Hocking, R. K.; Wasinger, E. C.; de Groot, F. M. F.; Hodgson, K. O.; Hedman, B.; Solomon, E. I. Journal of the American Chemical Society 2006, 128, 10442-10451.

(4) Hocking, R. K.; Wasinger, E. C.; Yan, Y.-L.; deGroot, F. M. F.; Walker, F. A.; Hodgson, K. O.; Hedman, B.; Solomon, E. I. Journal of the American Chemical Society 2007, 129, 113-125.

(5) Glatzel, P.; Bergmann, U. Coordination Chemistry Reviews 2005, 249, 65-95.

(6) Lundberg, M.; Kroll, T.; DeBeer, S.; Bergmann, U.; Wilson, S. A.; Glatzel, P.; Nordlund, D.; Hedman, B.; Hodgson, K. O.; Solomon, E. I. Journal of the American Chemical Society 2013, 135, 17121-17134.

(7) Kroll, T.; Hadt, R. G.; Wilson, S. A.; Lundberg, M.; Yan, J. J.; Weng, T.-C.; Sokaras, D.; Alonso-Mori, R.; Casa, D.; Upton, M. H.; Hedman, B.; Hodgson, K. O.; Solomon, E. I. Journal of the American Chemical Society 2014, 136, 18087-18099.

(8) Westre, T. E.; Kennepohl, P.; DeWitt, J. G.; Hedman, B.; Hodgson, K. O.; Solomon, E. I. Journal of the American Chemical Society 1997, 119, 6297-6314.

(9) Ballhausen, C. J. Introduction to Ligand Field Theory; McGraw-Hill Book Company, 1962.

(10) Arrio, M.-A.; Rossano, S.; Bruder, C.; Galoisy, L.; Calas, G. Europhysics Letters 2000, 51, 454-460. 
(11) Vercamer, V.; Hunault, M. O. J. Y.; Lelong, G.; Haverkort, M. W.; Calas, G.; Arai, Y.; Hijiya, H.; Paulatto, L.; Brouder, C.; Arrio, M.-A.; Juhin, A. Physical Review B 2017, $94,245115$.

(12) Hunault, M.; Vercamer, V.; Haverkort, M. W.; Arrio, M.-A.; Brouder, C.; Calas, G.; Juhin, A. Journal of Physics: Conference Series 2016, 721, 012005.

(13) Hunault, M. O. J. Y.; Khan, W.; Minar, J.; Kroll, T.; Sokaras, D.; Zimmermann, P.; Delgado-Jaime, M. U.; de Groot, F. M. F. Inorganic Chemistry 2017, 56, 10882-10892.

(14) deBeer George, S.; Brant, P.; Solomon, E. I. Journal of the American Chemical Society 2005, 127, 667-674.

(15) Baik, M.; Newcomb, M.; Friesner, R.; Lippard, S. Chemical Reviews 2003, 103, 23852419.

(16) Meunier, B.; de Visser, S. P.; Shaik, S. Chemical Reviews 2004, 104, 3947-3980.

(17) Huang, X.; Groves, J. T. Chemical Reviews 2018, 118, 2491-2553.

(18) Solomon, E. I.; Light, K. M.; Liu, L. V.; Srnec, M.; Wong, S. D. Accounts of Chemical Research 2013, 46, 2725-2739.

(19) Jasniewski, A. J.; Que, L., Jr. Chemical Reviews 2018, 118, 2554-2592.

(20) Park, K.; Li, N.; Kwak, Y.; Srnec, M.; Bell, C. B.; Liu, L. V.; Wong, S. D.; Yoda, Y.; Kitao, S.; Seto, M.; Hu, M.; Zhao, J.; Krebs, C.; Bollinger, J. M., Jr.; Solomon, E. I. Journal of the American Chemical Society 2017, 139, 7062-7070.

(21) Armstrong, W. H.; Lippard, S. J. Journal of the American Chemical Society 1984, 106, $4632-4633$.

(22) Armstrong, W. H.; Lippard, S. J. Journal of the American Chemical Society 1983, 105, $4837-4838$. 
(23) Lippard, S. J.; Schugar, H. J.; Walling, C. Inorganic Chemistry 1967, 6, 1825-1831.

(24) Schugar, H.; Walling, C.; Jones, R. B.; Gray, H. B. Journal of the American Chemical Society 1967, 89, 3712-3720.

(25) Sokaras, D.; Weng, T.-C.; Nordlund, D.; Alonso-Mori, R.; Velikov, P.; Wenger, D.; Garachtchenko, A.; George, M.; Borzenets, V.; Johnson, B.; Rabedeau, T.; Bergmann, U. Review of Scientific Instruments 2013, 84, 053102.

(26) Glatzel, P.; Bergmann, U.; Yano, J.; Visser, H.; Robblee, J. H.; Gu, W.; de Groot, F. M. F.; Christou, G.; Pecoraro, V. L.; Cramer, S. P.; Yachandra, V. K. Journal of the American Chemical Society 2004, 126, 9946-9959.

(27) Cowan, R. D. The Theory of Atomic Structure and Spectra; University of California Press: Berkeley, 1981.

(28) Butler, P. H. Point Group Symmetry: Applications, Methods and Tables; Plenum Press: New York, 1981.

(29) Thole, B. T.; van der Laan, G.; C.Fuggle, J.; Sawatzky, G. A.; Karnatak, R. C.; Esteva, J.-M. Physical Review B 1985, 32, 5107-5118.

(30) de Groot, F. M. F.; Kotani, A. Core Level Spectroscopy of Solids; CRC Press, Boca Raton, FL, 2008.

(31) Neese, F. Wiley Interdisciplinary Reviews: Computational Molecular Science 2012, 2, 73-78.

(32) Dirac, P. A. M. Proceedings of the Cambridge Philosophical Society 1930, 26, 376.

(33) Weigend, F.; Ahlrichs, R. Physical Chemistry Chemical Physics 2005, 7, 3297.

(34) Weigend, F. Physical Chemistry Chemical Physics 2006, 8, 1057.

(35) Neese, F. Inorganica Chimica Acta 2002, 337, 181. 
(36) DeBeer George, S.; Petrenko, T.; Neese, F. The Journal of Physical Chemistry A 2008, 112, 12936-12943.

(37) DeBeer-George, S.; Petrenko, T.; Neese, F. Inorganica Chimica Acta (60th birthday issue of Prof. E.I. Solomon) 2008, 361, 965-972.

(38) Chandrasekaran, P.; Stieber, S. C. E.; Collins, T. J.; Que, Jr., L.; Neese, F.; DeBeer, S. Dalton Transactions 2011, 40, 11070-11079.

(39) Roemelt, M.; Beckwith, M. A.; Duboc, C.; Collomb, M.-N.; Neese, F.; DeBeer, S. Inorganic Chemistry 2012, 51, 680-687.

(40) Rees, J. A.; Martin-Diaconescu, V.; Kovacs, J. A.; DeBeer, S. Inorganic Chemistry 2015, 54, 6410-6422.

(41) van Lenthe, E.; Baerends, E. J.; Snijders, J. G. The Journal of Chemical Physics 1993, $99,4597$.

(42) Wullen, J. V. The Journal of Chemical Physics 1998, 109, 392.

(43) Bernadotte, S.; Atkins, A. J.; Jacob, C. R. Journal of Chemical Physics 2012, 137, 204106.

(44) List, N. H.; Jensen, J. K. T. S. H. J. A.; Norman, P. Journal of Chemical Physics 2015, 142, 244111.

(45) Sørensen, L.; Guo, M.; Lindh, R.; Lundberg, M. Molecular Physics 2017, 115, 174-189.

(46) Ray, K.; DeBeer-George, S.; Solomon, E. I.; Wieghardt, K.; Neese, F. Chemistry - A European Journal 2007, 13, 2783.

(47) Mulliken, R. S. The Journal of Chemical Physics 1955, 23, 1833-1840.

(48) Tenderholt, A. L. QMForge: Tools for Converting the Output from QM Calculations into Something More Useful. 02-15-2016; http://qmforge. sourceforge .net. 
(49) Hocking, R. K.; DeBeer George, S.; Raymond, K. N.; Hodgson, K. O.; Hedman, B.; Solomon, E. I. Journal of the American Chemical Society 2010, 132, 4006-4015.

(50) de Groot, F. M. F. Chemical Reviews 2001, 101, 1779-1808.

(51) Kroll, T.; Aristov, V. Y.; Molodtsova, O. V.; Ossipyan, Y. A.; Vyalikh, D. V.; Büchner, B.; Knupfer, M. Journal of Physical Chemistry A 2009, 113, 8917-8922.

(52) Thole, B.; van der Laan, G. Physical Review A 1988, 38, 1943.

(53) Kurian, R.; Kunnus, K.; Wernet, P.; Butorin, S. M.; Glatzel, P.; de Groot, F. M. F. Journal of Physics: Condensed Matter 2012, 24, 452201.

(54) Krause, M. O.; Oliver, J. H. Journal of Physical and Chemical Reference Data 1979, 8,329 .

(55) de Groot, F. M. F.; Krisch, M. H.; Vogel, J. Physical Review B 2002, 66, 195112.

(56) George, S. D.; Brant, P.; Solomon, E. I. Journal of the American Chemical Society 2005, 127, 667-674.

(57) Guo, M.; Sørensen, L. K.; Delcey, M. G.; Pinjari, R. V.; Lundberg, M. Physical Chemistry Chemical Physics 2016, 18, 3250-3259.

(58) Vankó, G.; de Groot, F. M. F.; Huotari, S.; Cava, R. J.; Lorenz, T.; Reuther, M. arXiv 2008, 0802.2744 .

(59) Glatzel, P.; Mironea, A.; Eeckhout, S. G.; Sikora, M.; Giuli, G. Physical Review B 2008, r7., 115133.

(60) Juhin, A.; de Groot, F.; Vanko, G.; Calandra, M.; Brouder, C. Physical Review B 2010, 81, 115115.

(61) Cabaret, D.; Bordage, A.; Juhin, A.; Arfaoui, M.; Gaudry, E. Physical Chemistry Chemical Physics 2010, 12, 5619. 
(62) Cutsail, G. E.; Blaesi, E. J.; Pollock, C. J.; Bollinger, J. M.; Krebs, C.; DeBeer, S. Journal of Inorganic Biochemistry 2020, 203, 110877.

(63) Yan, J. J.; Kroll, T.; Baker, M. L.; Wilson, S. A.; Decreau, R.; Lundberg, M.; Sokaras, D.; Glatzel, P.; Hedman, B.; Hodgson, K. O.; Solomon, E. I. Proceedings of the National Academy of Sciences 2019, 116, 2854âĂŞ2859.

(64) Skulan, A. J.; Brunold, T. C.; Baldwin, J.; Saleh, L.; Bollinger, J. M., Jr.; Solomon, E. I. Journal of the American Chemical Society 2004, 126, 8842-8855.

(65) Jasniewski, A. J.; Komor, A. J.; Lipscomb, J. D.; Que, L., Jr Journal of the American Chemical Society 2017, 139, 10472-10485. 
Graphical TOC Entry

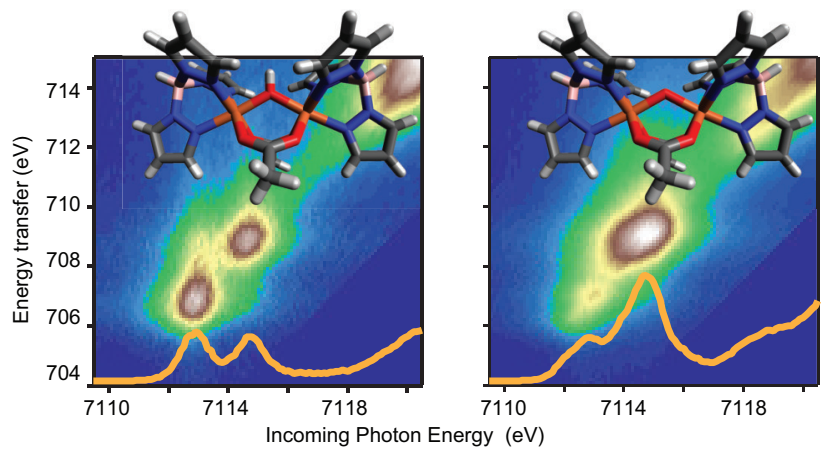

